\title{
EL OSO NEGRO AMERICANO EN EL NOROESTE DE MÉXICO: RECUPERACIÓN DE REGISTROS DE OCURRENCIA
}

\author{
Christian Alejandro Delfín-Alfonso, a, b* Carlos Alberto LóPez-GonzÁlez ${ }^{\text {a }}$ \\ Nalleli Elvira LARA-DíAZ ${ }^{\mathrm{a}}$ \\ ${ }^{a}$ Facultad de Ciencias Naturales, Universidad Autónoma de Querétaro, Av. de la Ciencia S/N \\ Juriquilla, Delegación Santa Rosa Jáuregui, Querétaro, Querétaro, México C. P. 76230. \\ C.E.: cada7305@gmail.com, cats4mex@aol.com,cuicuiri_milochomil@hotmail.com \\ *Dirección de contacto: C. Delfín-Alfonso C. E.: cada7305@gmail.com \\ Tel.: (442) 1921200 Ext.: 5341 \\ ${ }^{b}$ Red Medio Ambiente y Sustentabilidad, Instituto de Ecología, A. C. Carretera antigua a Coatepec \\ 351, El Haya, Xalapa 91070, Veracruz, México. C. E: christian.alejandro@inecol.edu.mx,
}

Tel-Fax: (228) 8421847 Ext.: 4328

Delfín-Alfonso, C. A., C. A. López-González \& N. E. Lara-Díaz. 2011. El oso negro americano en el noroeste de México: recuperación de registros de ocurrencia. Acta Zoológica Mexicana (n. s.), 27(3): 777-801.

RESUMEN. El conocimiento sobre la distribución de la mayoría de las especies es incompleto y fragmentado, debido a la falta de registros en colecciones científicas u observaciones fidedignas, como es el caso del oso negro americano (Ursus americanus) particularmente al NO de México. El objetivo fue recuperar datos históricos y actuales de ocurrencia de la especie en la ecorregión de la Sierra Madre Occidental y las Islas del Cielo. Se recopilaron registros de diversas colecciones y museos (nacionales y extranjeras), estudios históricos y actuales (artículos científicos, informes, tesis, reportes sin publicar) e informantes clave. Se obtuvieron 88 registros $(10 \hat{\jmath}, 10 \stackrel{+}{ }, 68$ sexo indeterminado) en un periodo de 120 años (1890-2010). De colecciones científicas se obtuvieron 54 registros, 29 fueron obtenidos de la literatura y cinco de informantes confiables. La mayoría de los registros se ubicaron espacialmente dentro de cinco Áreas Naturales Protegidas y seis Regiones Terrestres Prioritarias. Los Estados de Chihuahua y Sonora contaron con más registros, colectados en el período de 2001-2010; para los periodos de 1921-1930 y 1941-1950 no se obtuvieron registros. El bajo número de registros obtenidos es reflejo de la falta de estudios a lo largo de la distribución de la especie en México, y la carencia de reportes formales sobre avistamientos, capturas o muertes de los animales a nivel local. El presente trabajo contribuye sustancialmente a identificar y examinar los vacios geográficos de información respecto a la especie, funcionando como línea base para la toma de decisiones, principalmente para aplicar técnicas y métodos que ayuden a seleccionar sitios prioritarios para su conservación. Es imperante reconocer la necesidad de implementar estrategias de manejo y conservación de la especie, a fin de poder mantenerla a largo plazo en nuestro país.

Palabras clave: Distribución, México, Oso negro, registros, Sierra Madre Occidental.

Recibido: 23/05/2011; aceptado: 01/08/2011. 
Delfín-Alfonso, C. A., C. A. López-González \& N. E. Lara-Díaz. 2011. The American black bear in northwestern Mexico: retrieval of occurrence records. Acta Zoológica Mexicana (n. s.), 27(3): $777-801$.

ABSTRACT. Knowledge about the distribution of most species is incomplete and fragmentary, due to few records in scientific collections and a lack of reliable observations. The American black bear (Ursus americanus) provides a good example, especially in NW Mexico. Our objective was to retrieve historical data and document current occurrence of the species in the Sierra Madre Occidental ecoregion and the Sky Islands. We consulted records in several collections and museums (national and foreign), historical and current studies (scientific articles, reports, theses, unpublished reports) and key informants. We documented 88 records $(10 \AA, 10$, , and 68 of unknown sex) in a period of 120 years $(1890-2010)$. Of the Scientific collections 54 records were obtained, 29 were obtained from the literature and five from reliable informants. Most records fall within five protected areas and six priority terrestrial regions. The states of Chihuahua and Sonora had more records collected in the period 2001-2010 and for the periods 1921-1930 and 1941-1950 records were not obtained. The low number of records available reflects the lack of studies over the distribution of the species in Mexico, and the lack of formal reports on the sighting, capture or killing of animals at the local level. We believe this work contributes substantially to identify and examine the geographic information gaps regarding the species, functioning as a baseline for decision making, mainly to apply techniques and methods that help in selecting priority sites for conservation. It is imperative to recognize the necessity to implement management and conservation of the species, in order to maintain it long-term in our country.

Key words: Black bear, conservation, distribution, Mexico, records, Sierra Madre Occidental.

\section{INTRODUCCIÓN}

Tradicionalmente, los análisis de biogeografía se basan en localidades individuales y registros puntuales, esto limita el adecuado conocimiento de la distribución de los taxones, ya que para la mayoría de las especies, se carece de un número suficiente de especímenes colectados o de observaciones fidedignas (Ponder et al. 2001). Así, a menudo el conocimiento al respecto es incompleto y fragmentario (Peterson 2001, Ponder et al. 2001). En este sentido, algunos autores han enfatizado la importancia de concentrar registros confiables de las especies, con el fin de construir mapas precisos de distribución geográfica, llenando vacíos de información y muestreo, aumentando con ello las potencialidades de análisis e interpretación biogeografca (Ponder 1999, Funk \& Richardson 2002). Estos mapas basados en modelos de distribución, generados a partir de registros, ayudan a definir estrategias de manejo y conservación de la biodiversidad (Funk \& Richardson 2002). Tal es el caso del oso negro americano (Ursus americanus Pallas 1780) en México, considerado como el Úrsido más ampliamente distribuido y uno de los carnívoros más grandes de Norteamérica y (Kennedy et al. 2002).

El oso negro americano, es el carnívoro de mayor tamaño en México y la única especie de Úrsido presente en el país, contando con tres subespecies (U. a. eremicus Merriam 1904, U. a. amblyceps Baird 1859 y U. a. machetes Elliot 1903; Hall 1981, Lariviére 2001). La especie ocupaba las regiones boscosas de los estados del NO y 
NE de México: Sonora, Chihuahua, Coahuila, Tamaulipas, Durango, Zacatecas, Sinaloa, San Luis Potosí, Jalisco, Nayarit y posiblemente Aguascalientes (Leopold 1959, Baker \& Green 1962, Hall 1981). Actualmente, la información sobre su distribución es escasa, pero se considera que se ha reducido hasta en un $80 \%$ (INE/SEMARNAP 1999), principalmente en relación con su cacería y envenenamiento durante el Siglo $\mathrm{XX}$, al ser percibida como una amenaza al ganado y a los cultivos (Medellín et al. 2005), y aprovechada por sus pieles y su grasa (Baker 1956, Leopold 1959). Adicionalmente, el incremento de la población humana y una pobre economía han contribuido a la pérdida de hábitat para el oso negro (Pelton et al. 1999).

La legislación mexicana la reconoce como una especie prioritaria para su conservación (INE/SEMARNAT 1999), sin embargo, solo una subespecie (U. a. eremicus) distribuida en la Sierra Madre Oriental en el NE de México, es considerada como en Peligro de Extinción por la NOM-059-SEMARNAT-2010 (SEMARNAT 2010). La subespecie $U$. a. machetes y aparentemente $U$. a. amblyceps se distribuyen en el NO de México (Hall 1981), en la Sierra Madre Occidental (SOMc) y las Islas del Cielo (SI), ecorregión donde poco se conoce de su distribución y en general de sus poblaciones, y por tanto su estado de conservación es alarmante, aún cuando no se considera como una subespecie bajo ninguna categoría de riesgo (SEMARNAT 2010).

No obstante el creciente interés por la especie, los proyectos de investigación han sido pocos y aislados, impidiendo contar, hasta el momento, con información precisa y detallada acerca de la situación de algunas de las sub-poblaciones en el territorio nacional. Para el caso de las subespecies de oso negro en el NO de México, existen pocos registros en colecciones científicas y algunos de ellos presentan problemas de referencia geográfica como condición persistente. Esto limita la generación de modelos biogeográficos confiables, ya que no existe una sistematización de los registros de la especie, aun en las colecciones científicas más completas.

Dicho esto y en atención a la creciente preocupación nacional por recuperar, proteger y conservar la diversidad biológica del país, y particularmente por especies prioritarias como el oso negro americano, el principal objetivo de esta contribución fue dar a conocer en un compendio geográfico, los registros históricos y actuales de oso negro (en un periodo de 120 años, de 1890 a 2010) en el NO de México, para responder a un amplio rango de preguntas de investigación y conservación a largo plazo.

\section{MATERIAL Y MÉTODOS}

Área de estudio. El área de estudio comprende la ecoregión SMOc y SI, en los desiertos Sonorense y Chihuahuense, enclavada en ocho estados del NO de México con una superficie de $221,078.68 \mathrm{~km}^{2}$ (Fig. 1). Se caracteriza por terrenos abruptamente escarpados y pendientes pronunciadas hacia el Pacífico y suaves hacia el altiplano del Chihuahuense y la Meseta Central. La elevación varía de 1050 a los 


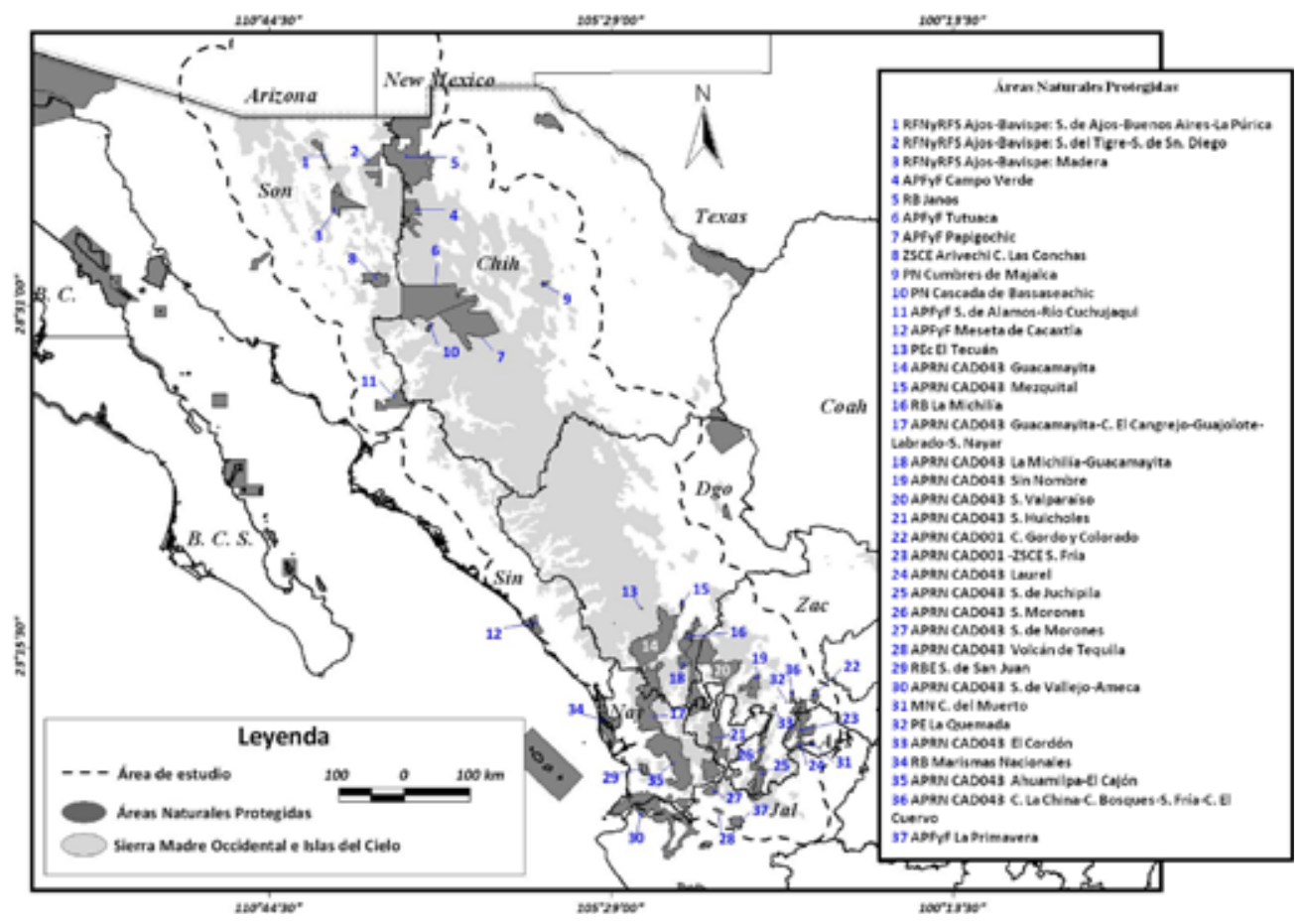

Figura 1. Delimitación del área de estudio donde se muestra la Sierra Madre Occidental, las SI y las ANP's en los ocho estados (RFNyRFS: Reserva Forestal Nacional y Refugio de Fauna Silvestre, APFyF: Área se Protección de Flora y Fauna Silvestre, ZSCE: Zona Sujeta a Conservación Ecológica-

Estatal, PN: Parque Nacional, APRN CAD: Área de Protección de los Recursos Naturales-Cuenca Alimentadora de Distrito de Riego, PE: Parque Estatal, PEc: Parque Ecológico-Estatal, RB: Reserva de la Biosfera, RBE: Reserva de la Biosfera Estatal).

3900 m.s.n.m. (Ferrusquia-Villafranca 1993), el clima es extremoso de templadocálido sub-húmedo en cimas con nevadas en las partes altas $\left(20^{\circ} \mathrm{C}\right.$ verano, $-20 \mathrm{C}^{\circ}$ invierno) a semiseco en los valles $\left(\sim 40^{\circ} \mathrm{C}\right.$ verano, $10^{\circ} \mathrm{C}$ invierno). La precipitación va de 500 a $1600 \mathrm{~mm}$ anuales con humedad relativa del 50-60 \% (García 1988). La vegetación dominante compuesta por elementos holárticos, domina sobre los neotropicales (Rzedowski 2006), es representada por bosques templados en las partes altas (bosques de: Pinus sp., Quercus sp., Juniperus sp., Cupressus sp., Picea sp., Pseudotsuga sp. y Abies sp.), bosques mixtos (Pinus-Quercus), bosque mesófilo de montaña en cañadas; matorral y pastizal en las partes bajas (crassicaule, desértico rosetófilo, micrófilo, submontano, mezquital-huizachal); en la vertiente pacífica se presentan selvas (caducifolias, subcaducifolias y espinosas), matorral subtropical y bosque bajo abierto (Palacio-Prieto et al. 2000, González-Elizondo et al. 2007). 
Recopilación de datos de ocurrencia. Durante 2009 y 2010, se consultaron colecciones científicas (nacionales y extranjeras) resguardadas en museos, instituciones de investigación y universidades (públicas y privadas), utilizando tres concentradores de bases de datos (Global Biodiversity Information Facility-http://www.gbif.org, Mammal Network Information System-http://www.manisnet.org y Arctos Multi-Institution-Multi-Collection-Museum Database-http://arctos.database.museum), para recopilar registros de ocurrencia en los últimos 120 años (1890-2010). Simultáneamente, se llevó a cabo una revisión de diversos recursos bibliográficos (tesis, libros, artículos científicos y de divulgación, reportes de agencias gubernamentales, entre otros), publicados en México y en el extranjero.

Con los registros obtenidos, se construyó una base de datos con información básica de colecta (longitud, latitud, código de colección, nombre de la institución, número de catálogo, estado, municipio, localidad, sexo del ejemplar, material depositado y/o tipo de registros, fecha de colecta, fuente y nombre del colector, entre otros datos). Un paso importante fue georreferenciar los registros que no tuvieran una localización espacial explicita con la ayuda de manuales de georreferenciación (Chapman \& Wieczorek 2006), gaceteros (BioGeomancer Working GroupC-http://www. biogeomancer.org, Geolocate, Tulane University-Museum of Natural History http:// www.museum.tulane.edu/geolocate/) y mapas del Instituto Nacional de Estadística, Geografía e Informática de México (http://www.inegi.org.mx).

Distribución espacio-temporal de registros. La base de datos se exportó a un manejador de Sistemas de Información Geográfica (ArcView ver. 3.2, ESRI 1999), para generar un mapa de distribución espacial de los registros. Los registros se agruparon cronológicamente a partir de 1890 hasta el 2010, esto se hizo con el fin de conocer la acumulación temporal de registros por unidad de tiempo, en este caso por décadas (1890-1900, 1901-1910, 1911-1920, 1921-1930, 1931-1940, 1941-1950, 1951-1960, 1961-1970, 1971-1980, 1981-1990, 1991-2000 y 2001-2010) a partir del registro más antiguo obtenido (1890) localizado en las bases de datos. Paralelamente, se utilizó un modelo digital de terreno obtenido del US Geological Survey's HYDRO1k dataset (USGS 2001) con una resolución espacial de $0.0083^{\circ}$ pixel $\left(\sim 1 \mathrm{~km}^{2}\right)$ para obtener la altitud de los registros.

Con el fin de conocer la distribución de los registros en los estados, municipios y áreas de conservación, los registros fueron ubicados sobre los siguientes mapas: municipios de México (INEGI 2009), Áreas Naturales Protegidas (ANP) de la Comisión Nacional de Áreas Naturales Protegidas de México (CONANP 2010), base de datos geográfica de Áreas Naturales Protegidas Estatales, del Distrito Federal y Municipales de México (Bezaury-Creel et al. 2009-CONABIO), Áreas Terrestres Protegidas de América del Norte (CEC 2010) y el mapa de Regiones Terrestres Prioritarias 
(RTP) de la Comisión Nacional para el Conocimiento y Uso de la Biodiversidad (CONABIO 2004). Con la información obtenida, se generaron histogramas de frecuencia para conocer su distribución por cada uno de los mapas utilizados, particularmente porque muchas de las ANP's establecidas en el Norte de México no son de reciente creación. Finalmente se recopilan los registros obtenidos en el Apéndice 1.

\section{RESULTADOS}

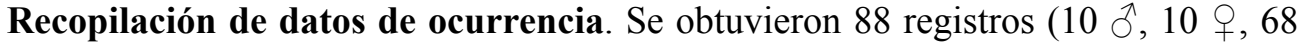
indeterminados) en un periodo de 120 años (1890-2010); 41 registros corresponden a ejemplares o parte de ellos depositados en alguna colección científica con número de catálogo asignado. Por otro lado, los registros no depositados en colección ( $\mathrm{n}=$ 47), 29 son reportados en la literatura y cinco más de comunicación personal con informantes del área de estudio (Cuadro 1, Apéndice 1). Es importante mencionar que durante la búsqueda de los registros, fue posible obtener la georreferencia del $95 \%$ $(n=84)$ de ellos, para los registros restantes $(n=4)$, no fue posible obtener espacialmente su localización (Apéndice 1) por carecer de datos específicos.

\section{Distribución espacio-temporal de los registros}

Registros por período de colecta. En los períodos comprendidos de 1890 a 1920 (1890-1900, 1901-1910, 1911-1920), fueron obtenidos 28 registros (31.5\% del total); para los períodos de 1921-1930 y 1941-1950 no se obtuvo ningún registro, y de 1951-1960 se obtuvieron registros solo en Chihuahua y Durango. En los períodos comprendidos de 1961 a 2000, se obtuvieron solo cinco registros. Finalmente, la mayoría de los registros obtenidos, se colectaron en el último período (2001-2010) y se distribuyen en el NO de la SMOc, entre Chihuahua y Sonora, cerca a la frontera con Estados Unidos (Apéndice 1, Fig. 2).

Registros por estados y municipios. Los estados con mayor número de registros son Chihuahua $(\mathrm{n}=47)$ y Sonora $(\mathrm{n}=31)$, que en conjunto comprenden el $88 \%$ de total. Para Durango se recopilaron siete registros (8\%). En la vertiente del Pacífico, particularmente para Sinaloa y Nayarit, solo se obtuvo un registro por estado y de Aguascalientes y Zacatecas no se obtuvieron datos de ocurrencia de la especie (Apéndice 1).

El área de estudio comprende 209 municipios distribuidos en los ocho estados, sin embargo, los registros obtenidos solo se ubican en 24 municipios. En los municipios de Chihuahua se cuenta con más registros $(\mathrm{n}=44)$, particularmente en Madera, Casas Grandes y Buenaventura. En Sonora se obtuvo un mayor número de registros dentro del municipio de Agua Prieta $(\mathrm{n}=22)$, seguido de Cumpas y Huachinera, con dos registros cada uno (Fig. 3). 
Cuadro 1. Fuentes de datos, número de registros y catálogo, obtenidos del Global Biodiversity Information Facility (http://www.gbif.org), del Mammal Network Information System (http://www. manisnet.org) y de Arctos Multi-Institution-Multi-Collection-Museum Database (http://arctos. database.museum/), de las colecciones de universidades y de bibliografía consultada.

\begin{tabular}{|c|c|c|c|}
\hline Fuentes de datos & Código & Registros & No. Catálogo \\
\hline $\begin{array}{l}\text { Centro de Investigación en Alimentación y Desarrollo, } \\
\text { A. C. Guaymas-Colección de Vertebrados }\end{array}$ & CIAD & 3 & $\begin{array}{l}210801-1,210801-2 \\
210801-3\end{array}$ \\
\hline $\begin{array}{l}\text { Comisión Nacional para el Conocimiento y Uso de la } \\
\text { Biodiversidad, Sistema Nacional de Información sobre } \\
\text { Biodiversidad (CONABIO) }\end{array}$ & SNIB & 4 & $\begin{array}{l}16863,49733 \\
60819,77697\end{array}$ \\
\hline $\begin{array}{l}\text { Universidad Autónoma de Querétaro, Lab. de Ecología } \\
\text { y Diversidad Faunística -Colección de Mamíferos }\end{array}$ & UAQ & 13 & $\begin{array}{l}\mathrm{S} / \text { número de } \\
\text { catálogo }\end{array}$ \\
\hline Field Museum Natural History-Mammals Collections & FMNH & 6 & $\begin{array}{l}19064,19012 \\
22362,89904 \\
89905,89906\end{array}$ \\
\hline $\begin{array}{l}\text { Instituto de Biología-Universidad Nacional Autónoma } \\
\text { de México-Colección Nacional de Mamíferos (CNMA) }\end{array}$ & IB & 1 & 1244 \\
\hline Michigan State University Museum (MSUM) & MR & 1 & 871 \\
\hline $\begin{array}{l}\text { University of California, Berkeley-Museum of } \\
\text { Vertebrate Zoology }\end{array}$ & MVZ & 4 & $\begin{array}{l}121819,121820 \\
121821,128494\end{array}$ \\
\hline $\begin{array}{l}\text { National Museum of Natural History (NMNH), } \\
\text { Smithsonian Institution-Vertebrate Zoology Mammals } \\
\text { Collections }\end{array}$ & USNM & 15 & $\begin{array}{c}99665,99338 \\
98329,98326, \\
98325,98322, \\
98321,203206, \\
177665,177664 \\
177663,177662 \\
177661,132195 \\
117100\end{array}$ \\
\hline $\begin{array}{l}\text { The Academy of Natural Sciences of Philadelphia- } \\
\text { Mammalogy Collection }\end{array}$ & ANSP & 4 & $\begin{array}{l}6881,6882,6883 \\
19995\end{array}$ \\
\hline Harvard University-Museum of Comparative Zoology & $\mathrm{MCZ}$ & 1 & 10502 \\
\hline $\begin{array}{l}\text { American Museum of Natural History (AMNH)- } \\
\text { Vertebrate Zoology Collection }\end{array}$ & M & 2 & 15867,21592 \\
\hline $\begin{array}{l}\text { Literatura (Allen } 1904 \text { \& 1906, Leopold 1958, Baker } \\
\text { \& Greer 1962, Anderson 1972, Trevino \& Jonkel 1986, } \\
\text { Vargas 1997, Verdugo 2005, Mendoza 2006, Sánchez- } \\
\text { Mateo et al. 2007, Moreno-Arzate 2007, Periódico El } \\
\text { Imparcial 2008, Lara-Díaz 2010) }\end{array}$ & $\mathrm{S} /$ datos & 29 & $\begin{array}{l}\mathrm{S} / \text { número de } \\
\text { catálogo }\end{array}$ \\
\hline $\begin{array}{l}\text { Comunicación personal (Carlos López-González, José } \\
\text { Hugo Martínez, Jorge Servín Martínez, Vidal Lozano) }\end{array}$ & $\mathrm{S} /$ datos & 5 & $\begin{array}{l}\text { S/ número de } \\
\text { catálogo }\end{array}$ \\
\hline Total & 88 & & \\
\hline
\end{tabular}




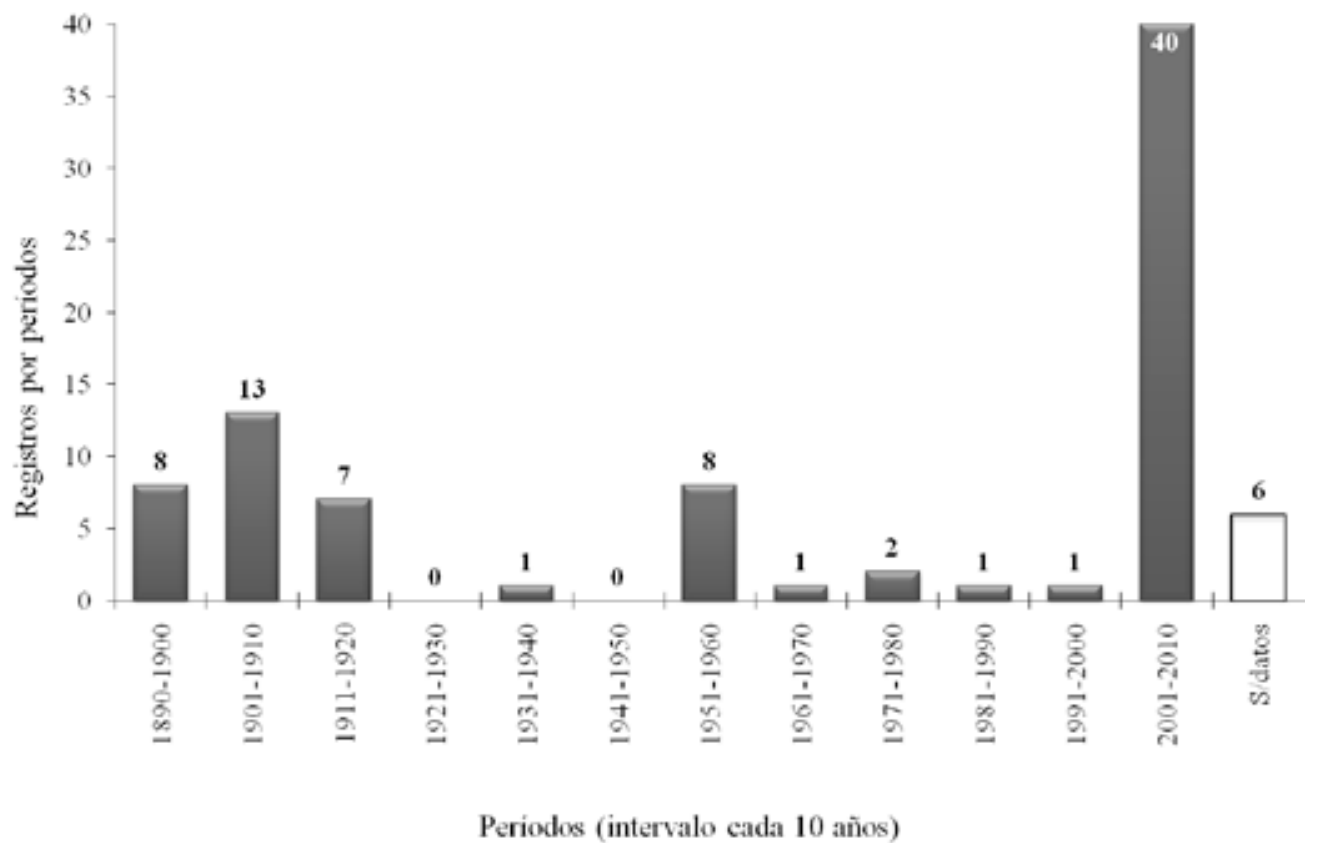

Figura 2. Agrupación de los registros obtenidos en períodos con intervalos de 10 años desde 1890 al 2010.

Registros en sitios importantes para la conservación. En el área de estudio, se localizaron 37 registros en lo que actualmente son ANP's con diferentes categorías de decreto y manejo, de las cuales, 28 tienen decreto federal y 11 con decreto estatal y municipal (Bezaury-Creel et al. 2009, CEC 2010, CONANP 2010). De los registros obtenidos, 20 fueron localizados en cinco ANP's, todas con decreto federal (Fig. 4). Por otro lado, se corroboró que 55 registros fueron localizados en seis de las 36 áreas consideradas en la actualidad como RTP's (Cuadro 2, Fig. 5), de estos registros, solo 19 cuentan con número de catálogo de depósito en alguna colección (Apéndice 1).

Distribución altitudinal de los registros. La mayoría de los registros se localizaron a una altitud por encima de los $1500 \mathrm{msnm}(84.3 \%, \mathrm{n}=74)$, cuatro registros no tienen georeferencia y no fue posible ubicarlos altitudinalmente, el resto de los registros $(\mathrm{n}=10)$ se localizaron por debajo de los $1500 \mathrm{msnm}$ (Fig. 6). El intervalo altitudinal de los registros oscila entre 1015 y $2809 \mathrm{msnm}$ (promedio $1980 \pm 396 \mathrm{msnm}$ ), siendo el registro del municipio El Rosario en Sinaloa (conocido como el registro de Escuinapa, Apéndice 1) el de menor altitud y el de Sierra de la Candela en Tepehuanes, Durango el de mayor altitud. 


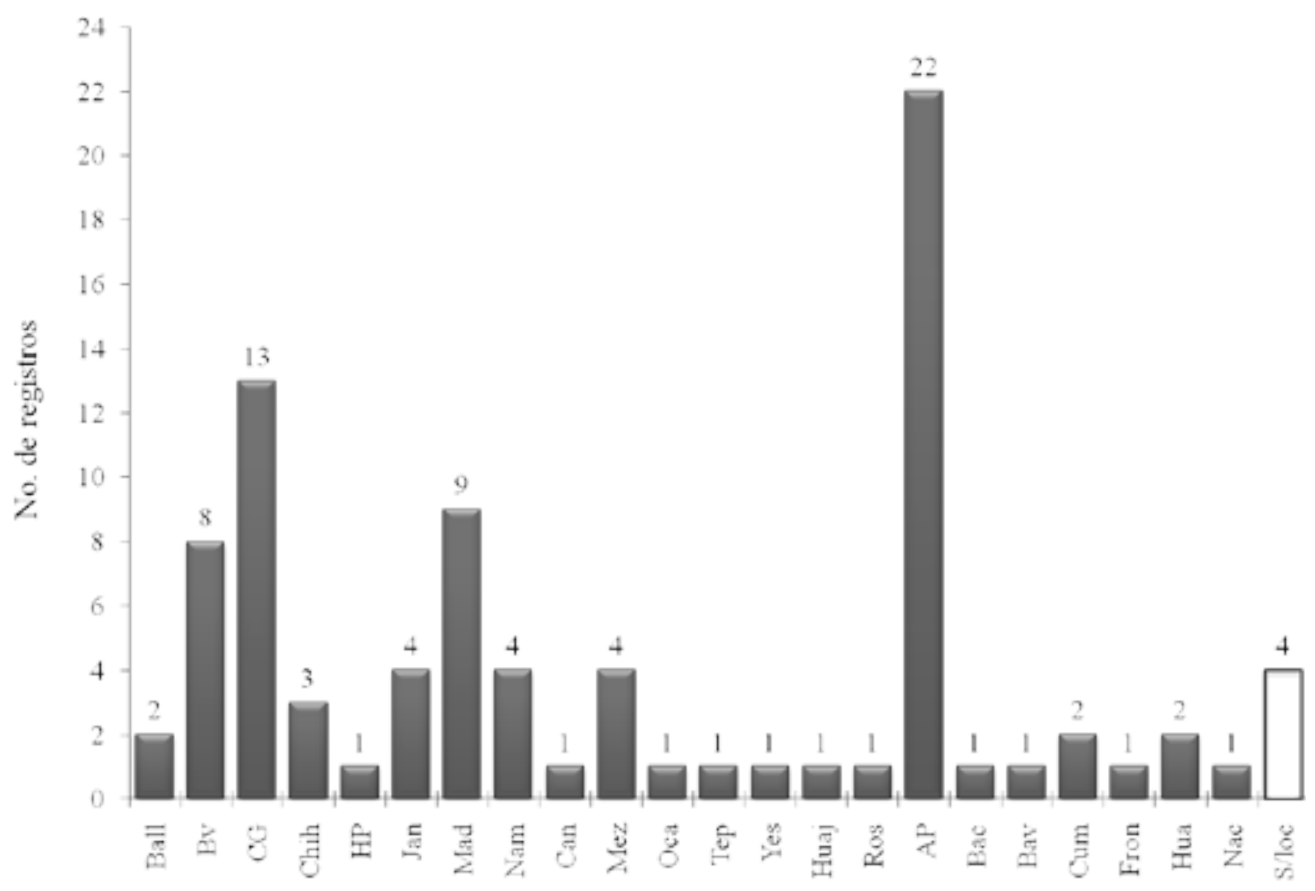

Municipios

Figura 3. Registros de Ursus americanus, distribuidos en el NO de México (CHIHUAHUA: BallBalleza, Bv-Buenaventura, CG-Casas Grandes, Chih-Chihuahua, HP-Hidalgo del Parral, Jan-Janos, Mad-Madera, Nam-Namiquipa; DURANGO: Can-Canatlán, Mez-Mezquital, Oca-Ocampo, TepTepehuanes; JALISCO: Yes-La Yesca; NAYARIT: Huaj-Huajicori; SINALOA: Ros-Rosario; SONORA: AP-Agua Prieta, Bac-Bacadéhuachi, Bav-Bavispe, Cum-Cumpas, Fron-Fronteras, HuaHuachinera, Nac-Nacozari de García, S/loc-Sin localidad).

Registros por detalle de preparación o tipo de registro. Los registros recuperados

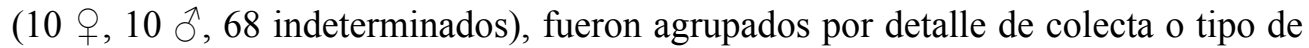
registro, los datos obtenidos muestran que existen 35 cráneos depositados en alguna colección (Cuadro 3, Fig. 7), sin embargo, un número considerable de registros son fotográficos $(n=16)$ recopilados en el último periodo (Cuadro 3$)$.

Por otra parte, se recopilaron cinco registros indeterminados (sin datos de colecta) de los que se obtuvo su referencia geográfica: registro de oso negro reportado a Leopold (1958) por lugareños en la cuenca del Río Bolaños entre Nayarit y Jalisco (probablemente en el municipio La Yesca, Nay.); dos registros en Arroyo del Nido en Chihuahua (Cat. SNIB-49733 y SNIB-60819) reportados por Ceballos (2002) y Ceballos et al. (2006), un registro de López Wilchis (1998) obtenido de la base de datos de CONABIO (Cat. SNIB-77697) y uno más de la base de datos del Instituto de 


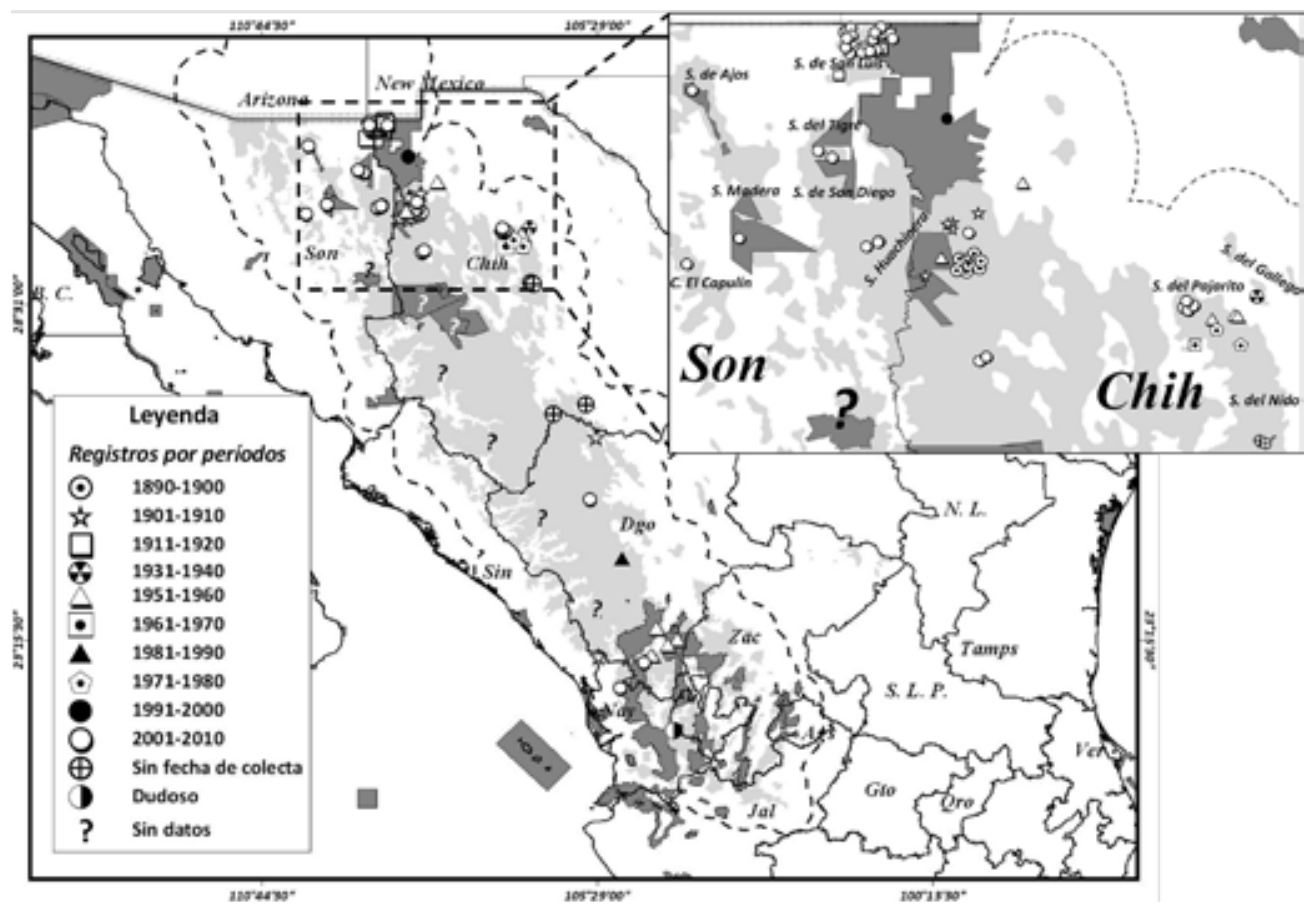

Figura 4. Distribución espacio-temporal de los registros de oso negro en la SMOc y las SI, combinado con las ANP's, los estados y agrupado en intervalos de 10 años.

Cuadro 2. Regiones Terrestres Prioritarias de CONABIO (2004) donde se ha registrado la presencia de Ursus americanus.

\begin{tabular}{lc}
\hline Nombre y Clave de RTP & No. de registros \\
\hline 42 Sierras Los Ajos-Buenos Aires-La Púrica & 1 \\
44 Bavispe-El Tigre & 10 \\
45 Sierra de San Luis-Janos & 27 \\
47 Sierra del Nido-Pastizal de Flores Magón & 14 \\
57 Guacamayita & 1 \\
59 Cuenca Río Jesús María & 2 \\
S/datos & 33 \\
Total & 88 \\
\hline
\end{tabular}




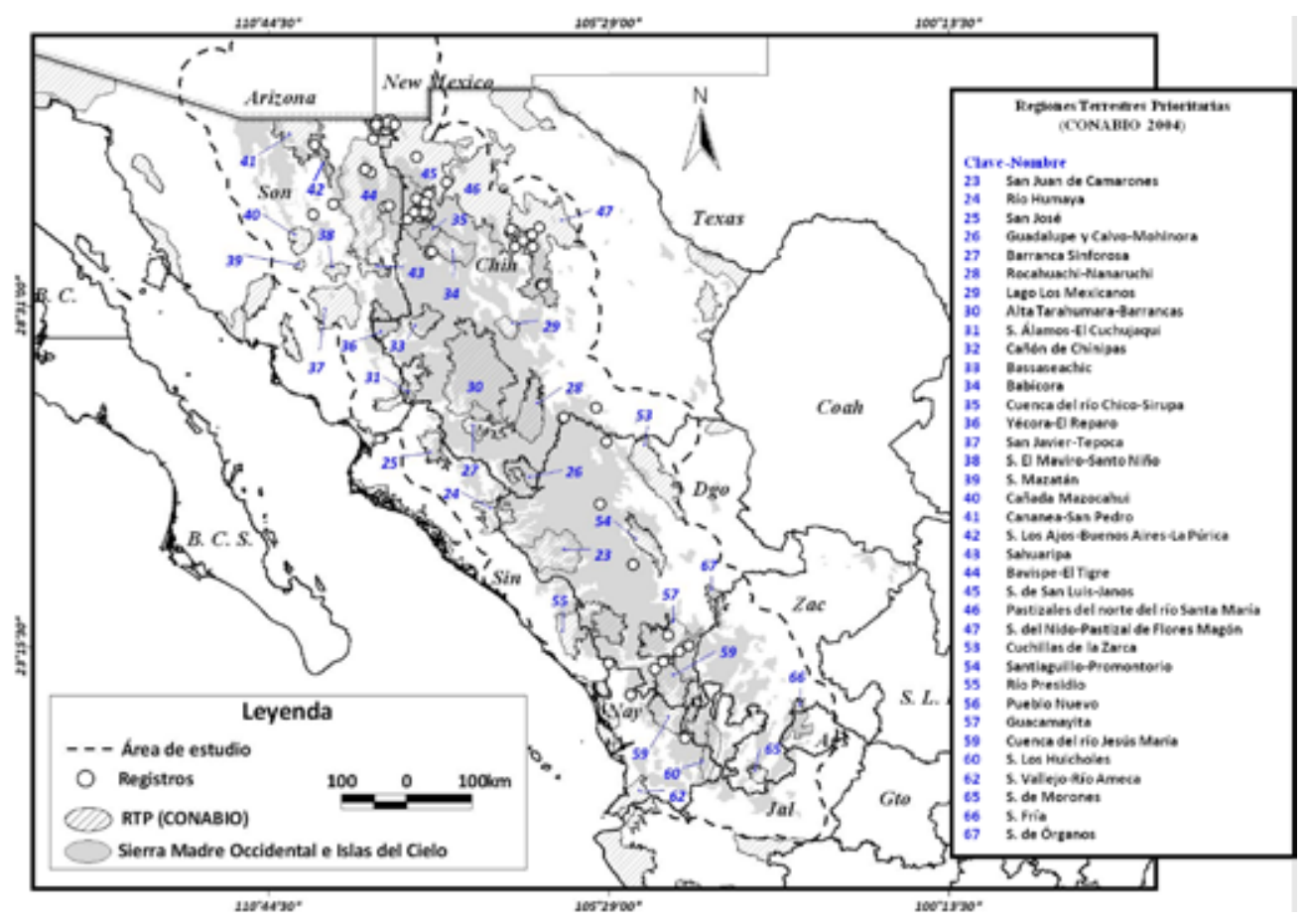

Figura 5. Registros de Ursus americanus en las RTP's que se incluyen en el área de estudio.

Biología-UNAM (Cat. CNMA-1244) en Casas Grandes, de este último, los datos de la etiqueta presenta un error en la identificación del estado, la localidad de registro se ubica sobre una zona urbana, muy cerca de Paquimé, Chihuahua. Durante la fase de recopilación, se obtuvo un registro de oso negro a $4 \mathrm{~km} \mathrm{~W}$ El Taiste, cerca de Huajicori en Nayarit (22/05/2007), se trata de una fotografía tomada en una zona de bosque de pino (reportan Vidal Lozano Meléndez, Fco. Javier Arámbula y Dagoberto Martínez Rosales, Consultoría Forestal y Ambiental Ing. Roberto Trujillo, Durango), la fotografía no está publicada en esta contribución por respetar los derechos de autor.

Cabe destacar el depósito en colección de cinco especímenes de los más completos en cuatro colecciones en los Estados Unidos, colectados en Chihuahua y Durango (Apéndice 1): Cat. ANSP-6881, ANSP-6883, FMNH-22362, USNM-98325 y MSUM-871 (espécimen voucher). Como información relevante, se obtuvo el registro de dos cachorros capturados por un cazador (fase alazán y otro negro) en un aserradero Tepehuano conocido como La China, junto al Rancho Las Margaritas, al SW de Vicente Guerrero, Durango, mismos que fueron puestos a la venta en junio de 1957 por $\$ 400$ (el par o $\$ 250$ pesos cada uno) en el mercado de la Cd. de Durango (Baker \& Greer 1962, Apéndice 1). 
Cuadro 3. Detalle de preparación o tipo de registro de Ursus americanus en el área de estudio.

\begin{tabular}{lc}
\hline Tipo & No. de registros \\
\hline Capturado & 1 \\
Cráneo & 21 \\
Cráneo \& esqueleto & 2 \\
Cráneo \& piel & 13 \\
Cráneo, piel \& báculo & 1 \\
Excreta & 14 \\
Excreta \& huella & 1 \\
Fotografía & 16 \\
Huella & 4 \\
Observado & 7 \\
Pelos & 1 \\
Piel & 1 \\
Piel, garras & 1 \\
Indeterminados & 5 \\
Total & 88 \\
\hline
\end{tabular}

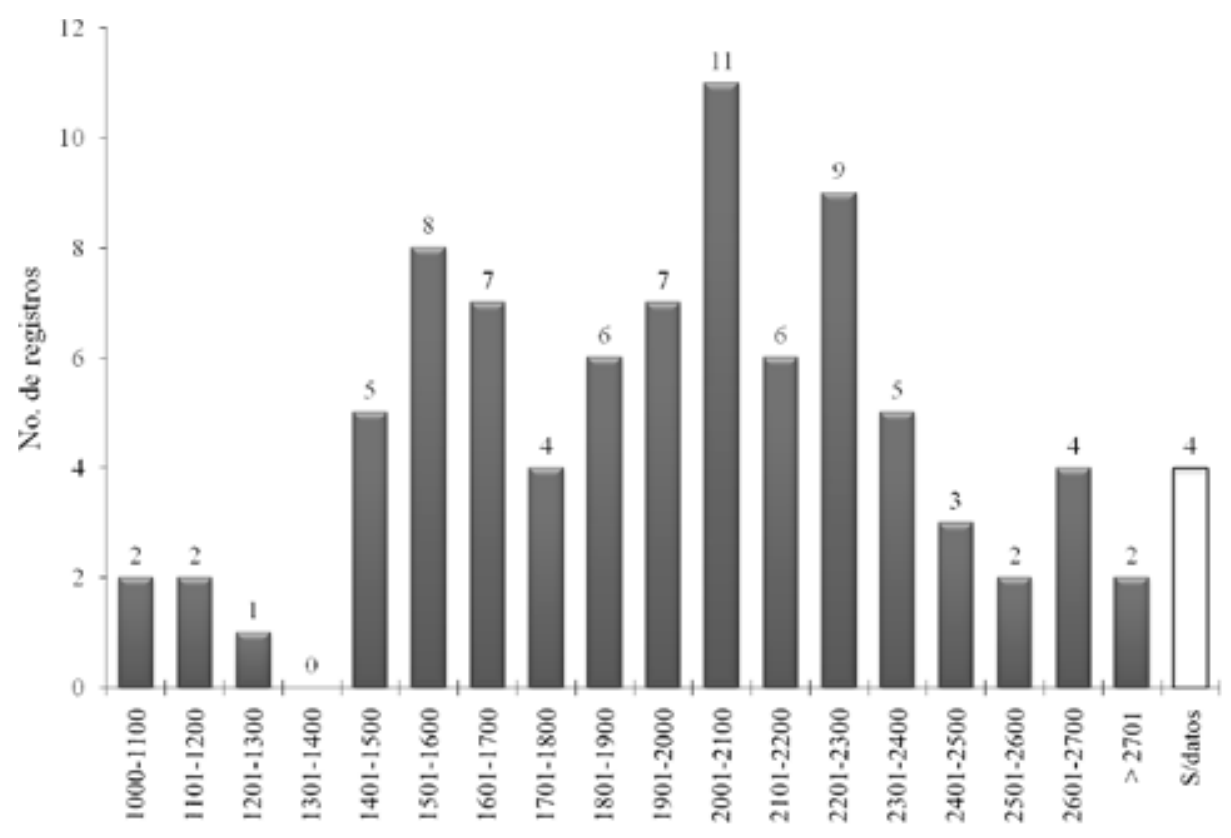

Altitud (msnm, intervalos $\mathrm{e} / 100 \mathrm{~m})$

Figura 6. Distribución altitudinal de los registros de Ursus americanus, obtenidos en al área de estudio. 


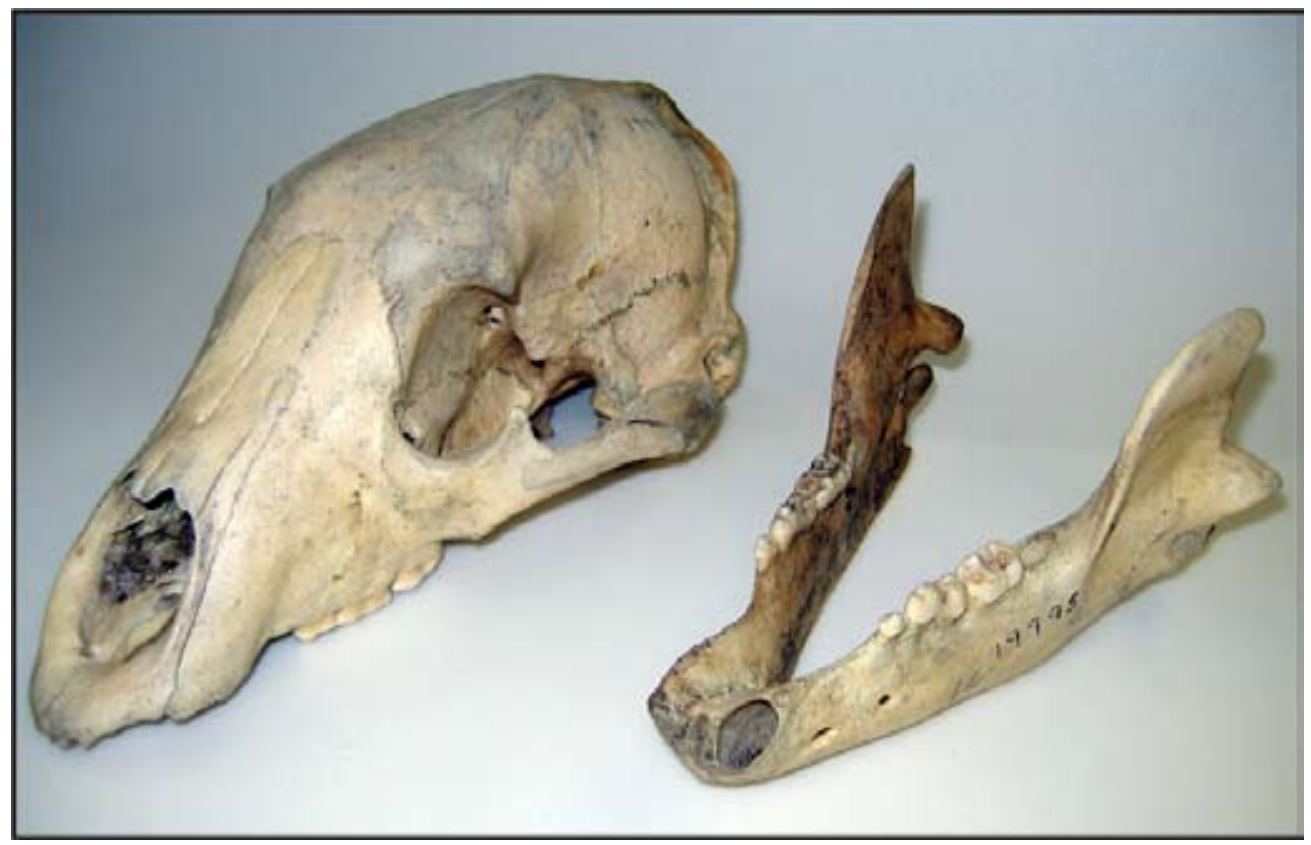

Figura 7. Cráneo de oso negro colectado por D. A. Feathers en Chihuahua y depositado en The Academy of Natural Sciences of Philadelphia-Mammalogy collection (datos de la etiqueta Cat. ANSP19995, Ursus americanus, 18 miles Southwest of Gallego, 6000 ft, Chihuahua, México, Dec. 2, 1936;

Cortesía del Dr. Ned Gilmore-administrador de la colección de la ANSP).

\section{DISCUSIÓN}

El número de registros de oso negro compilados en el presente estudio, se estima son pocos considerando que cubre un amplio periodo en años (120 años) en una superficie correspondiente al $11.26 \%\left(221,078.68 \mathrm{~km}^{2}\right)$ del territorio nacional y en términos relativos, los registros de oso negro obtenidos $(n=88$ en 120 años $)$ en el área de estudio, son similares a los obtenidos para los jaguares $(\mathrm{n}=78)$ en un periodo de 100 años obtenidos en Sonora y Chihuahua (Brown \& López-González, 2001).

Los registros se obtuvieron para cinco de los ocho estados del área de estudio, destacando Sonora y Chihuahua. Seguramente, existen algunos trabajos de investigación (p. ej. tesis, reportes no publicados, otros textos informales), donde han sido registrados osos negros, principalmente en el estado de Chihuahua, sin embargo, durante la búsqueda de información resultó complicada la obtención de los mismos. Esta situación posiblemente si refleja la carencia de estudios a lo largo de la distribución de la especie en México, y la escasez de reportes formales sobre avistamientos, capturas o muertes de los animales a nivel local. Un factor que puede influir en esto, es que el oso negro no es considerado como un trofeo de caza (Carmony 1998) en el NO 
de México, en comparación con especies como el jaguar (Brown \& López-González 2001), por lo que cuando son cazados (p. ej. por supuesta depredación de ganado), sus pieles, cráneos o esqueletos son generalmente desechados, esta situación ocasiona la pérdida de registros por parte de los habitantes de la región.

Temporalmente, solo cinco de las 12 décadas o períodos, presentaron más de cinco registros cada período. Existen varios factores científicos, políticos y sociales que pudieron influir en la distribución temporal de los registros. La inestabilidad político-social por el movimiento revolucionario y post-revolucionario por el que transitaba México de 1910 a 1930 (Foweraker \& Craig 1990), pudo provocar la ausencia de naturalistas en esa época y por ende la falta de registros de la especie. Esto sin duda se corrobora con el desarrollo histórico de la mastozoología formal en México, ya que los estudios en mastozoología renacen lentamente en la década de 1930 correspondiente a la post-revolución y su renacimiento formal como disciplina a partir de 1940 (Guevara-Chumacero et al. 2001).

Un periodo con más registros es de 1951-1960, corresponde a una época de máxima exploración por parte de investigadores norteamericanos en México (Leopold 1958, Baker \& Greer 1962, Anderson 1972). Posterior a 1960 y hasta principios de 1990, los datos que existen pueden relacionarse a la ejecución de la campaña de erradicación de depredadores (Division PARC-Predatory Animal and Rodent Control) en la unión americana y norte de México (Villa 1960, Brown 1984 \& 1996, Hawthorne 2004). Esta situación provocó vacios de información en la línea de tiempo y la pérdida invaluable de registros de la especie, pero por otro lado, existieron compilaciones de registros relacionados con la eficiencia de las técnicas antidepredadores para otros carnívoros (p. ej. uso de veneno 1080; Villa 1960, Molina-Bravo 1964).

En décadas recientes los registros aumentan, aunado a un mayor conocimiento e interés sobre la pérdida de especies y degradación de ecosistemas, por lo que instituciones educativas, de investigación y federales han desarrollado, implementado y fortalecido programas de monitoreo y conservación de especies (p. ej. Programa de Conservación de Especies en Riesgo, CONANP 2007).

Regionalmente, el área con más registros recientes es la Sierra de San Luis, Sonora, lo que puede indicar que la población de osos negros en esta región muestra indicios de una posible recuperación, además de ser la más grande del NO de México y en su caso, es donde actualmente se están llevando a cabo algunas investigaciones con la especie (López-González \& Lara-Díaz 2010). En el resto de las SI en México, se ha documentado la presencia osos negros en la RFN y RFS Ajos Bavispe, cuya poligonal se encuentra fragmentada en varios polígonos, separada por ranchos privados (CONANP 2003), haciendo incierto el estado poblacional de los osos. Cabe mencionar, que en otras ANP's como la R. B. La Michilía (Dgo.), P. N. Basasseachic, APFyF Tutuaca, APFyF Papigochic (Chih.) y APRN CAD001-ZSCE Sierra Fría (Ags.), no se obtuvieron registros verificables, sin embargo, anecdóticamente se 
conoce que los osos negros estuvieron presentes en el siglo pasado al igual que el lobo mexicano (Com. Pers. Maurilio Anchondo-CONANP, Abraham de Alba-INIFAP Ags., José Ángel Moreno-R. B. La Michilía; Maury 1993, Tinker 1978).

En los estados de Chihuahua, Durango, Aguascalientes y Zacatecas, existen grandes vacíos de información, reflejado en la carencia de registros. Los registros aislados entre Nayarit y Durango, son un síntoma del posible proceso de recolonización de estas áreas. Actualmente muchos registros se han obtenido en Agua Prieta, Sonora y se debe al interés de instituciones de investigación, agencias gubernamentales y ONG's, que desarrollan proyectos de investigación en la Sierra de San Luís y la RFN-RFS Ajos-Bavispe. Los registros actuales obtenidos en la frontera norte entre Chihuahua, Sonora, Arizona y New Mexico, se añaden a los obtenidos por naturalistas y cazadores norteamericanos (p. ej. Ben Lylli, E. W. Nellson, E. A. Goldman y R. L. Hardin) a principios del siglo pasado $(\sim 1900)$, acostumbrados a cazar en la frontera Norte (Carmony 1998) depositaban los ejemplares o parte de ellos en el NMNH-Smithsonian Institution.

La carencia de registros formales de oso negro en otras áreas del NO de México, ha limitado la evaluación de la conectividad, tendencias y estado poblacional de la especie. Al respecto, existe un vacío geográfico donde no se han registrado osos negros (al menos sistemáticamente), esta área comprende alrededor de $100000 \mathrm{~km}^{2}$ al NO de Durango y SO de Chihuahua ( 28 municipios, Fig. 4) entre el parte aguas de la SMOc y la vertiente del Pacífico y tal vez, la falta de registros en esta zona es por la falta de prospecciones de campo y no a la ausencia de la especie.

\section{Conclusiones e implicaciones de manejo}

La situación del oso negro en México es incierta, particularmente en las subpoblaciones del área de estudio, donde el panorama general es la falta de conocimiento ecológico de la especie, por lo que resulta necesario influir en la percepción social de las comunidades, y generar interés hacia el oso negro, no como una especie de caza, sino como una especie funcional dentro de los ecosistemas, que finalmente influye en las actividades productivas de la región.

La distribución potencial del oso negro en México se presenta en un área muy extensa, por lo que resulta complicada la recopilación de los registros. La base generada en el presente estudio es clave para la utilización de diversas herramientas que nos permitan restringir la búsqueda, como los modelos basados en la probabilidad de ocupación y detección de la especie (MacKenzie et al. 2003) y modelos de distribución potencial basados en registros puntuales. En este sentido, la presente contribución ayuda sustancialmente a identificar y examinar los vacios geográficos de información respecto a la especie.

La identificación de esta brecha de muestreo o laguna de información, proporciona información sobre cómo plantear preguntas para realizar nuevos estudios, en los 
sitios en los que no se tiene conocimiento de la especie. Al respecto fueron reconocidos 185 municipios que se localizan en la SMOc, donde es recomendable dirigir futuros estudios y verificar la posible presencia o ausencia (histórica y actual) de la especie, de entre los que destacan por contener sierras con bosques templados en su interior: CHIHUAHUA-Urique, Nonoava, Morelos, Maguarichi, Guazapares, Guadalupe y Calvo, Guachochi, Carichí, Bocoyna, Batopilas; DURANGO-Topia, Tepehuanes, Tamazula, Santiago Papasquiaro, San Dimas, Pueblo Nuevo, Otáez, Guanaceví, Canelas; Súchil y Mezquital; JALISCO-Mezquitic (municipio Huichol); NAYARIT-Del Nayar y, ZACATECAS-Valparaíso, Monte Escobedo, Jiménez del Teúl y Chalchihuites, entre otros.

A otra escala espacial de análisis, se identificaron ANP's donde se presume la especie está o estuvo presente, donde no se tienen registros puntuales y se caracterizan por la presencia de bosques templados de coníferas y mixtos, adecuados para la especie, por lo tanto, es recomendable promover proyectos de investigación en: APFyF Tutuaca, APFyF Papigochic, ZSCE Arivechi Cerro Las Conchas, PN Cascada de Bassaseachic, APFyF Sierra de Álamos-Rio Cuchujaqui, Porciones de las APRN-CADNR043 y 01 (Guacamayita-Cerro El Cangrejo-Guajolote-Labrado-Sierra Nayar, Sierra Valparaíso, La Michilía-Guacamayita, Sierra Huicholes, Sierra Fría, Sierra de Morones y Cerro La China-Cerro Bosques-Sierra Fría-Cerro El Cuervo) entre otras áreas no menos importantes (ver Figuras 1 y 4 ).

Contar con registros que permitan conocer y entender los patrones de distribución y dispersión del oso negro en su área de distribución, e inferir el estado de sus subpoblaciones, ayudará a mantener su viabilidad, considerando además que la conectividad con las poblaciones hacia Estados Unidos puede favorecer la recuperación de la especie. Por último, es indispensable reconocer la necesidad de instrumentar estrategias de manejo y conservación de la especie, a fin de poder mantenerla a largo plazo; por lo tanto, esta información, sirve como línea base para la toma de decisiones, principalmente para la aplicación de diversas técnicas y métodos que ayuden a seleccionar sitios prioritarios para su conservación.

AGRADECIMIENTOS. Agradecemos a las siguientes personas por su apoyo y su valiosa ayuda al M. en C. Heli Coronel Arellano, Dr. Abraham De Alba Ávila, Dr. José H. Martínez Guerrero, Dr. Jorge I. Servín Martínez, Dr. Ned Gilmore (Academy of Natural Sciences of Philadelphia-Mammalogy Collection) y a la Dra. Laura Abraczinskas (Michigan State University Museum) y a tres revisores anónimos por sus acertadas apreciaciones. Este trabajo obtuvo apoyo financiero parcial de la Universidad Autónoma de Querétaro, Instituto de Ecología, A. C., The International Association for Bear Research and Management (IBA), Fondos Mixtos CONACYT-Gobierno del Estado de Querétaro (CONACyT-QRO2008-CO1-96464), Naturalia, A.C., Fondo Mexicano para la Conservación de la Naturaleza, Instituto Nacional de Ecología (INE), Comisión Nacional de Áreas Naturales Protegidas (CONANP) a través de la RFN y RFS Ajos Bavispe y la Comisión Nacional para el Conocimiento y Uso de la Biodiversidad (CONABIO). 


\section{LITERATURA CITADA}

Allen, J. A. 1904. Further notes on mammals from northwestern Durango. Bulletin of the American Museum of Natural History, 20: 205-210.

Allen, J. A. 1906. Mammals from the states of Sinaloa and Jalisco, Mexico, collected by J. H. Batty during 1904 and 1905 Batty Expeditions to Mexico 1903-1906. Bulletin of the American Museum of Natural History, 22: 191-262.

Anderson, S. 1972. Mammals of Chihuahua taxonomy and distribution. Bulletin of the American Museum of Natural History, 48: 149-410.

Armstrong, D. M., J. Knox Jones, Jr. \& E. C. Birney. 1972. Mammals from the Mexican State of Sinaloa. III. Carnivora and Artiodactyla. Journal of Mammalogy, 53: 48-61.

Baker, R. H. 1956. Mammals of Coahuila, Mexico. Univ. Kansas Publ., Museum of Natural History, 59: 125-335.

Baker, R. H. \& J. K. Greer. 1962. Mammals of the Mexican state of Durango. Museum of Michigan State University Biology Series 2: 29-159.

Bezaury-Creel, J. E., J. F. Torres, L. M. Ochoa-Ochoa, M. Castro \& N. Moreno. 2009. Base de Datos Geográfica de Áreas Naturales Protegidas Estatales, del Distrito Federal y Municipales de México, Versión 2.0, (Julio 31, 2009). TNC-CONABIO-CONANP (On line: http://www.conabio. gob.mx/informacion/gis/).

Brown, D. E. \& C. A. López González. 2001. Borderland jaguars: Tigres de la frontera. Salt Lake City: The University of Utah Press.

Carmony, N. B. 1998. Ben Lilly's tales of bears, lions and hounds. High-Lonesome Books, NM, USA. 212 pp.

Ceballos, G. 2002. Actualización de la base de datos del Atlas Mastozoológico de México. Universidad Nacional Autónoma de México. Instituto de Ecología. Bases de datos SNIB2010-CONABIO Proyectos No. T009 y A003. México, D. F.

Ceballos, G., S. Blanco, C. González \& E. Martínez. 2006. Distribución potencial de Ursus americanus oso negro americano. Extraído del Proyecto DS006 "Modelado de la distribución de las especies de mamíferos de México para un análisis GAP”. Instituto de Biología, UNAM-CONABIO (On line: http://www.conabio.gob.mx/informacion/gis/).

CEC. 2010. North American Environmental Atlas-Terrestrial Protected Areas of North America 2010. Scale 1:10,000,000 Vector digital data. Department of Forestry and Natural Resources, Clemson University for the Commission for Environmental Cooperation in Agreement with CONANP-Mexico, CARTS-Canada, Quebec-MDDEP \& USGS. Montreal, Québec, Canada (On line http://www. cec.org/naatlas).

Chapman, A. D. \& J. Wieczorek. 2006. Guide to Best Practices for Georeferencing. Copenhagen: Global Biodiversity Information Facility. 90 pp.

CONABIO. 2004. Regiones Terrestres Prioritarias. Comisión Nacional para el Conocimiento y Uso de la Biodiversidad-CONABIO. Escala 1:1000000. México (On line: http://www.conabio.gob.mx/ informacion/gis/).

CONANP. 2003. Estudio previo justificativo "Área de Protección de Flora y Fauna Mavavi, Hermosillo, Sonora”. Comisión Nacional de Áreas Naturales Protegidas-SEMARNAT. México.

CONANP. 2007. Programa de conservación de especies en riesgo. PROCER. 2007-20012. En: http:// www.conanp.gob.mx/pdf_especies/PROCERFinalpubmar2009.pdf.

CONANP. 2010. Áreas Naturales Protegidas Federales de México. Serie 1, Escala 1:50,000 y 1:250,000. Comisión Nacional de Áreas Naturales Protegidas-SEMARNAT (On line: http://www.conanp.gob. $\mathrm{mx} / \mathrm{sig} /$ informacion/info.htm). 
ESRI. 1999. Arc View ver. 3.2. Environmental Systems Research Institute, Inc. Redlands, California, USA.

Ferrusquia-Villafranca, I. 1993. Geology of Mexico-A synopsis. Pp. 3-107. In: Ramamoorthy, T.P., R. Bye, A. Lot \& J. Fa (ed.). Biological diversity of Mexico: Diversity and Distribution. Oxford University Press, New York.

Foweraker, J. \& A. L. Craig. 1990. Popular movements and political change in Mexico. Lynne Rienner Publishers, Colorado, USA. 314 pp.

Funk, V. A. \& K. S. Richardson. 2002. Systematic data in biodiversity studies: use it or lose it. Systematic Biology, 51: 303-316.

Gallo-Reynoso, J. P., T. Van Devender, A. L. Reina-Guerrero, J. Egido-Villarreal \& E. Pfeiler. 2008. Probable occurrence of a brown bear Ursus arctos in Sonora, Mexico, in 1976. The Southwestern Naturalist, 532: 256-260.

García, E. 1988. Modificaciones al sistema Köppen-García (para adaptarlo a las condiciones de la República Mexicana). Offset Larios. México, D. F.

González-Elizondo, M. S., M. González-Elizondo \& M. A. Márquez-Linares. 2007. Vegetación y Ecorregiones de Durango. Plaza \& Valdez Press, Instituto Politécnico Nacional, CIIDIR-Durango, Dgo., México.

Guevara-Chumacero, L., R. López-Wilchis y V. Sánchez-Cordero. 2001. 105 años de investigación mastozoológica en México (1890-1995): una revisión de sus enfoques y tendencias. Acta Zoológica Mexicana (n. s.) 83: 35-72.

Hall, E. R. 1981. The mammals of North America. 2nd ed. Vol. 2. John Willey and Sons. NY.

Hawthorne, D. W. 2004. The history of federal and cooperative animal damage control. Sheep \& Goat Research Journal, 19: 13-15.

INE/SEMARNAT. 1999. Proyecto para la Conservación y Manejo del Oso Negro (Ursus americanus) en México. Instituto Nacional de Ecología-Secretaría de Medio Ambiente y Recursos Naturales, Gobierno Federal, México, 105 pp.

INEGI. 2009. Marco Geoestadístico Municipal 2009 Versión 4.1. Conjunto de datos vectoriales del Marco Geoestadístico Nacional. Instituto Nacional de Estadística, Geografía e Informática. Escala 1:250 000 (On line: http://mapserver.inegi.org.mx/).

Kennedy, M. L., Kennedy, P. K., Bogan, M. A. \& Waits, J. L. 2002. Geographic Variation in the Black Bear (Ursus americanus) in the Eastern United States and Canada. The Southwestern Naturalist, 47: 257-266.

Lara-Díaz, N. 2010. La comunidad de mamíferos previa a la liberación de Canis lupus baileyi en Sonora, México. Tesis de Maestría en Ciencias. Facultad de Ciencias Naturales. Universidad Autónoma de Querétaro. Querétaro, México, 123 pp.

Lariviére, S. 2001. Ursus americanus. Mammalian Species, 647: 1-11.

Leopold, A. S. 1958. Situación del Oso Plateado en Chihuahua. Revista de la Sociedad Mexicana de Historia Natural, 19: 115-120.

Leopold, A. S. 1959. Wildlife of Mexico. Berkeley and Los Angeles, Univ. California Press, xiii +568 pp.

López-Wilchis, R. 1998. Base de datos de mamíferos de México depositados en colecciones de Estados Unidos y Canadá. Universidad Autónoma Metropolitana. Unidad Iztapalapa. Bases de datos SNIBCONABIO. Proyecto No. P130. México, D.F.

López-González, C. A. \& N. E. Lara Díaz. 2010. Diagnóstico poblacional del oso negro (Ursus americanus) en las serranías de los estados de Sonora y Chihuahua y sus posibles afectaciones por el muro fronterizo. Informe Final. Instituto Nacional de Ecología- Universidad Autónoma de Querétaro. México. 94 pp. 
MacKenzie, D. I., J. D. Nichols, J. E. Hines, M. G. Knutson \& A. B. Franklin. 2003. Estimating site occupancy, colonization, and local extinction when a species is detected imperfectly. Ecology, 84: 2200-2207.

Maury, M. E. 1993. La Michilía Encuesta. En: A. Gómez-Pompa \& R. Dirzo. (eds.). Proyecto de evaluación de las áreas naturales protegidas de México. SEDESOL. México.

Medellin, R. A., C. Manterola, M. Valdez, D. G. Hewitt, D. Doan-Crider \& T.E. Fulbright. 2005. History, Ecology, and Conservation of the Pronghorn Antelope, Bighorn Sheep, and Black Bear in Mexico. Pp. 387-404 In Jean-Luc E. Cartron, G. Ceballos \& R. Stephen Felger (eds.). Biodiversity, ecosystems, and conservation in northern Mexico. Oxford University Press, NY

Mendoza, G. 2006. Diversidad de mamiferos terrestres de dos ecosistemas del municipio de Casas Grandes, Chihuahua, México. Tesis de Licenciatura. Universidad Autónoma de Ciudad Juárez, Cd. Juárez, Chihuahua, México.

Molina-Bravo, R. 1964. Control de animales depredadores del ganado en el estado de Sonora. Tesis Licenciatura. Universidad Nacional Autónoma de México, México, 22 pp.

Moreno-Arzate, C. N. 2008. Ecología conductual del oso negro (Ursus americanus) en la Sierra Madre Occidental. Tesis de Maestría, Instituto de Ecología, A. C., Veracruz, México.

Pacheco, J., G. Ceballos \& R. List. 2000. Los Mamíferos de la región de Janos-Casas Grandes, Chihuahua, México. Revista Mexicana de Mastozoología, 4: 69-83.

Palacio-Prieto, J. L., Bocco, G., Velásquez, A., J. F. Mas, F. Takaki, A. Victoria, L. Luna, G. Gómez, J. López, M. Palma, I. Trejo, A. Peralta, J. Prado, A. Rodríguez, R. Mayorga \& F. González. 2000. La condición actual de los recursos forestales en México: resultados del inventario forestal nacional 2000. Boletín del Instituto de Geografia, 43: 183-203.

Pelton, M. R., A. B. Coley, T. H. Eason, D. L. Doan Martinez, J. A. Pederson, F. T. Van Manen \& K. M. Weaver. 1999. American black bear conservation action plan. Pp. 144-156 In: C. Servheen, S. Herrero \& B. Peyton (Comps.). Bears. Status survey and conservation action plan. IUCN/ SSC bear and polar bear specialist groups. IUCN, Gland, Switzerland and Cambridge, UK.

Peterson, A. T., M. A. Ortega-Huerta, J. Bartley, V. Sanchez-Cordero, J. Soberon, R. H. Buddemeier \& D. R. B. Stockwell. 2002. Future projections for Mexican faunas under global climate change scenarios. Nature, 416: 626-629.

Peterson, T. A. 2001. Predicting species' geographic distributions based on ecological niche modeling. The Condor, 103: 599-605.

Ponder, W. F. 1999. Using museum collection data to assist in biodiversity assessment. Pp. 253-256. In: W. F. Ponder \& D. Lunney (Eds.). The Other 99\%: The conservation and biodiversity of invertebrates. Royal Zoological Society of New Gales, Mosman, NSW.

Ponder, W. F, G. A. Carter, P. Flemons \& R. R. Chapman. 2001. Evaluation of museum collection data for use in biodiversity assessment. Conservation Biology, 153: 648-657.

Rzedowski, J. 2006. Vegetación de México. $1^{a}$ Edición Digital. Comisión Nacional para el Conocimiento y Uso de la Biodiversidad (CONABIO), México. (Online: http://www.conabio.gob.mx/institucion/centrodoc/doctos/vegetacion_de_mexico.html)

SEMARNAT. 2010. Norma Oficial Mexicana NOM-059-SEMARNAT-2010, Protección ambiental-Especies nativas de México de flora y fauna silvestres-Categorías de riesgo especificaciones para su inclusión, exclusión o cambio-lista de especies en riesgo. Diario Oficial de la Federación. Segunda Sección. 30 de Diciembre de 2010. México.

Tinker, B. 1978. Mexican Wilderness and Wildlife. University of Texas Press, Austin. 131 pp.

Treviño, J. C. \& C. Jonkel. 1986. Do Grizzly Bears Still Live in Mexico? International Conference on Bear Research and Management, 6: 11-13. 
USGS. 2001. HYDRO 1k, elevation derivative database. United States Geological Survey, Sioux Falls, South Dakota. (Online: http://www.edcdaac.usgs.gov/gtopo30/hydro).

Vargas, F. 1997. Parques Nacionales de México. Aspectos fisicos, sociales, legales, administrativos, recreativos, biológicos, culturales, situación actual y propuestas en torno a los Parques Nacionales de México. Unidad Coordinadora de Áreas Naturales Protegidas. Instituto Nacional de Ecología. México. 720 pp.

Verdugo-Valenzuela, D. 2005. Caracterización del hábitat de oso negro (Ursus americanus machetes) en la Sierra El Pajarito en Chihuahua, México. Facultad de Zootecnia, Universidad Autónoma de Chihuahua, Chihuahua, México. 59 pp.

Villa, B. 1960. Combate contra lobos y coyotes del Norte de México. Anales del Instituto de Biología, México, 31:463-499.

\section{APÉNDICE 1}

Registros de oso negro reportados en este trabajo, desde 1890 al 2010. Se narran por orden cronológico y estado, incluyendo fecha (día/mes/año), sitio de colecta (localidad y municipio), sexo ( $q=$ hembra, $ठ$ $=$ macho), detalle del registro (tipo de registro o parte del espécimen depositado), colector, número de catalogo, altitud (msnm), tipo de vegetación (tipo de vegetación según el Inventario Nacional Forestal), la fuente del registro (colección o referencia bibliográfica) y notas u observaciones generales. Las colecciones consultadas fueron: Comisión Nacional para el Conocimiento y Uso de la Biodiversidad (CONABIO-SNIB), Centro de Investigación en Alimentación y Desarrollo, A. C. (CIAD), Field Museum Natural History (FMNH), Instituto de Biología-UNAM-Colección Nacional de Mamíferos (CNMA-IB), Michigan State University Museum (MSUM-MR), University of California, Berkeley (MVZ), National Museum of Natural History-Smithsonian Institution (USNM), The Academy of Natural Sciences of Philadelphia, (ANSP), Harvard University (MCZ), Americam Museum of Natural History (AMNH-M), Universidad Autónoma de Querétaro (UAQ, no disponible al público), Secretaría de Desarrollo Urbano y Ecología-Gobierno Federal Mexicano (SEDUE, no disponible al público). Literatura consultada que se utilizó: Allen (1904 \& 1906), Leopold (1958), Baker \& Greer (1962), Anderson (1972), Treviño \& Jonkel (1986), Vargas (1997), Verdugo (2005), Mendoza (2006), Moreno-Arzate (2007), Sánchez-Mateo et al. (2007), Periódico El Imparcial (2008), Gallo-Reynoso et al. (2008), Lara-Díaz 2010, LópezGonzález \& Lara Díaz 2010). Las coordenadas de los registros, en su mayoría, pueden ser consultadas directamente en las colecciones a través de su web, el resto, se encuentra bajo resguardo de los autores.

\section{Chihuahua (47 registros)}

00/00/00, Parte alta Arroyo Las Hadas, P. N. Cumbres de Majalca, Chihuahua, Observado, 2137 msnm, bosque de pino-encino (incluye encino-pino), (Vargas 1997). Reportado sin localización geográfica exacta para el P. N. Cumbres de Majalca, solo mencionan el paraje.

00/00/00, Rinconada de Álamos, P. N. Cumbres de Majalca, Chihuahua, Observado, 2114 msnm, bosque de pino-encino (incluye encino-pino), (Vargas 1997). Reportado sin localización geográfica exacta para el P. N. Cumbres de Majalca, solo mencionan el paraje

00/00/00, Arroyo del Nido, Balleza, $2006 \mathrm{msnm}$, pastizal natural (incluye pastizal-huizachal), (SNIB60819, CONABIO-SNIB, Ceballos 2002). Registros obtenidos de la base de datos de CONABIO del Proyecto T009.

00/00/00, Arroyo del Nido, Balleza, $2090 \mathrm{msnm}$, pastizal natural (incluye pastizal-huizachal), (SNIB49733, CONABIO-SNIB, Ceballos 2002). Registros obtenidos de la base de datos de CONABIO del Proyecto T009.

00/00/00, Indeterminada, Hidalgo del Parral, 1773 msnm, (SNIB-77697, López Wilchis 1998). Registro obtenido de CONABIO del Proyecto P130, la localidad se sobrepone a la zona urbana de Hidalgo 
de Parral, tal vez por el registro puede proviene de la Sierra de Los Azules (3 km W de Hidalgo de Parral) en una zona de bosque mixto seco (encino-pino).

0/0/1899, Colonia Garcia, Madera, đ̊, Cráneo, H. A. Cluff, 2401 msnm, bosque de pino, (USNM-99665, NMNH database).

15/7/1899, Colonia Garcia, Casas Grandes, Ô, Cráneo \& piel, E. W. Nelson \& E. A. Goldman, 2524 msnm, bosque de pino (USNM-98321, NMNH database).

23/7/1899, Colonia Garcia, Madera, Ô, Cráneo \& piel, E. W. Nelson \& E. A. Goldman, 2397 msnm, pastizal inducido (USNM-98322, NMNH database).

1/8/1899, Colonia Garcia, Madera, Ô, Cráneo, piel \& báculo, E. W. Nelson \& E. A. Goldman, 2296 msnm, bosque de pino (USNM-98325, NMNH database).

4/8/1899, Colonia Garcia, Casas Grandes, , Cráneo \& piel, E. W. Nelson \& E. A. Goldman, 2612 ms$\mathrm{nm}$, bosque de pino-encino (incluye encino-pino), (USNM-98326, NMNH database).

11/8/1899, Colonia Garcia, Madera, + , Cráneo, E. W. Nelson \& E. A. Goldman, 2240 msnm, bosque de pino-encino (incluye encino-pino), (USNM-98329, NMNH database).

18/8/1899, Colonia Garcia, Madera, ㅇ, Cráneo \& piel, E. W. Nelson \& E. A. Goldman, 2399 msnm, bosque de pino, (USNM-99338, NMNH database).

18/9/1899, Colonia Garcia, Casas Grandes, ๆ, Cráneo, C. H. Barber, 2524 msnm, bosque de pino, (M15867, AMNH database).

0/0/1901, Casas Grandes, Casas Grandes, Cráneo, C. L. Sanford, 2314 msnm, bosque de pino-encino (incluye encino-pino), (FMNH-19012, FMNH database).

0/0/1901, Casas Grandes, Casas Grandes, Cráneo \& piel, C. L. Sanford, 2314 msnm, bosque de pinoencino (incluye encino-pino), (FMNH-19064, FMNH database).

0/0/1901, Sierra Madre, Casas Grandes, Madera, Cráneo, C. L. Sanford, 1614 msnm, bosque de encino, (FMNH-89904, FMNH database).

0/0/1902, Chihuahua near Colonia Garcia, Casas Grandes, Cráneo \& piel de un juvenil, H. A. Cluff, $2278 \mathrm{msnm}$, bosque de pino-encino (incluye encino-pino), (ANSP-6881, ANSP database).

0/0/1902, Chihuahua, near Colonia Garcia, Casas Grandes, Cráneo \& piel, H. A. Cluff, 2278 msnm, bosque de pino-encino (incluye encino-pino), (ANSP-6882, ANSP database).

0/0/1902, Chihuahua, near Colonia Garcia, Casas Grandes, Cráneo \& piel de un subadulto, H. A. Cluff, $2278 \mathrm{msnm}$, bosque de pino-encino, (ANSP-6883, ANSP database).

0/2/1902, Colonia Garcia, Casas Grandes, Cráneo, H. A. Cluff, 2612 msnm, bosque de pino-encino (incluye encino-pino), (MCZ-10502, MCZ database).

0/3/1902, Colonia Garcia, Casas Grandes, , , Cráneo, H. A. Cluff, 2278 msnm, bosque de pino-encino (incluye encino-pino), (USNM-117100, NMNH database).

0/9/1903, Colonia Garcia, Casas Grandes, †, Cráneo, J. H. Gaut, 2278 msnm, bosque de pino-encino (incluye encino-pino), (USNM-132195, NMNH database).

0/0/1907, Cráneo \& piel, montado (Cat. Tax. \# 1494), msnm, (FMNH-22362, Anderson 1972).

0/0/1907, Indeterminada, Indeterminado, Cráneo, F. M. Stephenson, (FMNH-89905, FMNH database).

0/0/1911, San Luis Mountains, Janos, + , Cráneo \& piel, $2057 \mathrm{msnm}$, bosque de pino-encino (incluye encino-pino), (USNM-177664, NMNH database). Tal vez se trate de un espécimen cazado por B. V. Lilly, debido al sitio de colecta, al número de catálogo y fecha.

13/5/1911, San Luis Mountains, 12 Mi S of U.S., Janos, Cráneo \& piel, B. V. Lilly, 1930 msnm, bosque de pino, (USNM-177661, NMNH database). Espécimen cazado por B. V. Lilly documentado por Carmony (1998).

0/0/1912, Indeterminada, Indeterminado, Cráneo, F. M. Stephenson, S/datos msnm, (FMNH-89906, FMNH database).

2/12/1936, 18 mi. SW Gallego, 6000 ft, Chihuahua, Cráneo, D. A. Feathers, 1998 msnm, pastizal natural (incluye pastizal-huizachal), (ANSP-19995, Anderson 1972). 
3/4/1953, Casas Grandes, Casas Grandes, A. Málaga, 1492 msnm, asentamiento humano, (CNMA1244, IBUNAM database). Los datos de la etiqueta tienen un error en la identificación del estado y la localidad de registro se ubica en una zona urbana cerca de Paquimé, Chihuahua.

16/6/1957, Hacienda Providencia, Sierra del Nido, Buenaventura, Piel \& garras, A. S. Leopold, $2700 \mathrm{msnm}$, bosque de pino-encino (incluye encino-pino), (MVZ-121821, MVZ UC database, Leopold 1958).

23/6/1957, Arroyo El Nido, 25 mi SW Gallego, Namiquipa, Cráneo, A. S. Leopold, 1762 msnm, pastizal natural (incluye pastizal-huizachal), (MVZ-121819, MVZ UC database, Leopold 1958).

23/6/1957, Arroyo El Nido, 25 mi SW Gallego, Buenaventura, Cráneo, A. S. Leopold, 1756 msnm, pastizal natural (incluye pastizal-huizachal), (MVZ-121820, MVZ UC database, Leopold 1958).

0/0/1958, 15 mi. of Colonia Garcia, Madera, Cráneo \& piel, 2184 msnm, bosque de pino, (Anderson 1972).

15/8/1961, Arroyo El Mesteño, Sierra El Nido, Namiquipa, Cráneo, William Z. Lidicker Jr., J. D. A, 2037 msnm, bosque bajo-abierto, (MVZ-128494, MVZ UC database).

0/5/1979, Cañón de Los Prieto en Sierra del Nido, Namiquipa, Pelos, J. Treviño \& C. Jonkel, 1972 ms$\mathrm{nm}$, bosque de pino-encino (incluye encino-pino), (Treviño \& Jonkel 1986). Individuo observado durante prospección de campo en busca de osos pardos en la Sierra del Nido.

0/5/1979, Ojo del Álamo en Sierra del Nido, Namiquipa, Observado, J. Treviño \& C. Jonkel, 2426 ms$\mathrm{nm}$, bosque de pino-encino (incluye encino-pino), (Treviño \& Jonkel 1986). Dos individuos (color canela y negro) observados, se estimo su peso en alrededor de $100 \mathrm{~kg}$, individuos observados durante prospección de campo en busca de osos pardos en la Sierra del Nido.

24/2/1995, Casa de Janos, Janos, Huella, J. Pacheco, $1471 \mathrm{msnm}$, pastizal natural (incluye pastizal-huizachal), (SNIB-16863, CONABIO-SNIB, Pacheco et al. 2000). Registros obtenidos de la base de datos de CONABIO Proyecto B043, publicados por Pacheco et al. (2000).

1/2/2004, Sierra El Pajarito, Rancho Santa Mónica, Buenaventura, Excreta, D. Verdugo, 2096 msnm, bosque de pino-encino (incluye encino-pino), (Verdugo-Valenzuela 2005).

1/2/2004, Sierra El Pajarito, Rancho Santa Mónica, Buenaventura, Excreta, D. Verdugo, 2096 msnm, bosque de galería, (Verdugo-Valenzuela 2005).

1/2/2004, Sierra El Pajarito, Rancho Santa Mónica, Buenaventura, Excreta, D. Verdugo, 2096 msnm, bosque de galeria, (Verdugo-Valenzuela 2005).

1/6/2004, Sierra El Pajarito, Rancho Santa Mónica, Buenaventura, Excreta, D. Verdugo, 2253 msnm, bosque de pino-encino (incluye encino-pino), (Verdugo-Valenzuela 2005).

1/6/2004, Sierra El Pajarito, Rancho Santa Mónica, Buenaventura, Excreta, D. Verdugo, 2000 msnm, bosque de pino-encino (incluye encino-pino), (Verdugo-Valenzuela 2005).

24/3/2005, Ejido Ignacio Zaragoza, Casas Grandes, Huella, G. Mendoza, $2100 \mathrm{msnm}$, bosque de encino, (Mendoza 2006).

1/8/2006, Ejido 5 mi. W Santuario Madera, Cd. Madera, Madera, Huella, 2696 msnm, bosque de pino, (Sánchez-Mateo et al. 2007). Registro de oso negro es una zona de anidación de cotorra serrana en Chihuahua.

1/8/2006, Ejido La Norteña cerca de Presa Peñitas, Madera, Excreta, M. A. Sánchez-Mateo, 2400 msnm, bosque de pino-encino (incluye encino-pino), (Sánchez-Mateo et al. 2007). Registro de oso negro es una zona de anidación de cotorra serrana en Chihuahua.

0/10/2007, Rancho Santa Mónica, Sierra del Nido, Buenaventura, Excreta \& huella, C. N. Moreno-Arzate, $2104 \mathrm{msnm}$, bosque de pino-encino (incluye encino-pino), (Moreno-Arzate 2007).

30/10/2010, Sierra de San Luis, Janos, Excreta, N. Lara-Díaz \& C. A. López-González, 2081 msnm, bosque de pino-encino (incluye encino-pino), (UAQ-S/Cat, UAQ database, Lopez-González \& Lara-Díaz 2010). Registros obtenidos de un proyecto de la UAQ, financiado por el Instituto Nacional de Ecología (INE). 


\section{Durango (7 registros)}

30/8/1903, Rio Ocampo, Ocampo, Cráneo, J. H. Batty, 1740 msnm, bosque bajo-abierto, (M-21592, AMNH database). Mencionado por Allen (1904); las coordenadas del gacetero de Baker \& Greer (1962) referente a esta localidad, colocan el registro a $1.2 \mathrm{~km}$ al NO de la zona urbana de Villa Ocampo, sobre matorral xerófilo muy cerca del Río Florido.

0/9/1956, 40 mi. S and 17 mi. W Vicente Guerrero, Arroyo Los Timones, Rancho Las Margaritas, Mezquital, đ̂, Cráneo \& esqueleto parcial (voucher), R. H. Baker, $2418 \mathrm{msnm}$, pastizal inducidoencinar, (MSU-871, MSUM database, Baker \& Greer 1962). El registro en Baker \& Greer (1962) aparece con la localidad $28 \mathrm{mi} \mathrm{S}$ and $17 \mathrm{mi} \mathrm{W}$ Vicente Guerrero, sin embargo, no es correcta la localidad. Los restos son de un macho (cráneo y una porción del esqueleto con dientes desgastados), cazado en septiembre de 1956 y regalado a R. Baker (RHB \# 3286, 7/07/1957) por Joel González encargado del Rancho Las Margaritas (información del Diario de Campo del Dr. Baker depositado en el MUSM y proporcionada por la Dra. Laura Abraczinskas, Collections Manager Vertebrate Collections of Michigan State University Museum).

25/6/1957, $24 \mathrm{mi} \mathrm{W}$ and $29 \mathrm{mi} \mathrm{S}$ of Vicente Guerrero, Mezquital, Excreta, J. Carrillo (cazador), 1907 msnm, bosque de pino-encino (incluye encino-pino), (Baker \& Greer 1962). La excreta encontrada por el Sr. Julián Carrillo en compañía de R. Baker, contenía trozos de bellotas, escarabajos y el pelo de mamíferos.

25/6/1957, Aserradero TepehuanoLa China,juntoal Rancho Las Margaritas, al SW Vicente Guerrero, Mezquital, Capturado, $2135 \mathrm{msnm}$, bosque de pino-encino (incluye encino-pino), (Baker \& Greer 1962). El registro corresponde a dos cachorros capturados (uno alazán y otro negro) que debían ser transportados a la ciudad de Durango para ponerlos a la venta en el mercado. El dueño de los cachorros comentó a Baker que esperaba recibir $\$ 400$ por el par o $\$ 250$ por cada uno, si se venden por separado.

0/0/1989, Paraje Quebrada de Media Noche, Canatlán, Piel, 2712 msnm, bosque de pino-manzanita, (Com. Pers. Dr. José Hugo Martínez G.-UJED). Se trata de una piel sin depósito en colección y se desconoce su paradero, cazado en 1989 y confiscada la piel por personal de SEDUE el mismo año.

0/0/2004, Sierra de la Candela, Tepehuanes, Tepehuanes, Observado, $2809 \mathrm{msnm}$, bosque de pino. (Com. Pers., Dr. Jorge I. Servín-UAM-X).

0/0/2005, Alrededor de Canoas, Sierra de Mezquital, Mezquital, Observado, 2192 msnm, bosque de pino-encino (Com. Pers., Dr. Jorge I. Servín-UAM-X).

\section{Jalisco-Nayarit (1 registro)}

00/00/00, Rio Bolaños, La Yesca, A. S. Leopold, 1100 msnm, pastizal inducido, (Leopold 1958). Menciona Leopold (1958 y 1959) la presencia de oso negro reportada por lugareños, en la cuenca del río Bolaños entre Nayarit y Jalisco, sin embargo la localización del registro es incierta, aun cuando en el mapa de Leopold (1959) el registro esta marcado.

\section{Nayarit (1 registro)}

22/5/2007, Aproximadamente 4 km W El Taiste, cerca de Huajicori, Huajicori, Fotografía, Vidal Lozano Meléndez, 1114 msnm, bosque de encino, (Com.-Pers., Vidal Lozano Meléndez, Fco. Javier Arámbula, Dagoberto Martínez Rosales-Consultoría Forestal y Ambiental del Ing. Roberto Trujillo).

\section{Sinaloa (1 registro)}

6/1/1904, Vicinity of Escuinapa (12 mi around), Rosario, Piel, J. H. Batty, $1015 \mathrm{msnm}$, bosque de pino-encino (incluye encino-pino), (Allen 1906). Representado por una piel imperfecta, que J. H. Batty compró a un cazador nativo en la primavera del 1904 en las proximidades de Escuinapa; la localidad es representada a $12 \mathrm{mi}$. al E de Escuinapa (Allen 1906), donde inician las estribaciones de la SMOc hacia Durango, por encima de los 1290 msnm (4000 ft). La evidencia más plausible 
es la aparición de encinos de gran tamaño a esta altitud y es en esa zona donde probablemente fue cazado el oso. Ese mismo año (19/04/1904) Batty obtuvo cercana a esta localidad, una piel completa de Ursus arctos, depositada en el AMNH (M-24690). El registro en Escuinapa ha sido polémico y la identificación como un oso negro es un supuesto en base a consideraciones geográficas (Armstrong et al. 1972).

\section{Sonora (31 registros)}

0/0/1911, ô, Cráneo, R. L. Hardin, (USNM-203206, NMNH database). Los datos de la etiqueta del registro en la base de datos del NMNH, aparece como espécimen de Sonora sin referencia geográfica más explícita.

0/0/1911, San Luis Mountains, Agua Prieta, đ̃, Cráneo \& piel, 1821 msnm, chaparral, (USNM-177665, NMNH database). Tal vez se trate de algún espécimen colectado por B. V. Lilly.

0/6/1911, San Luis Mountains, Agua Prieta, ô, Cráneo, Lilly, B. V., 1859 msnm, bosque de pino-encino (incluye encino-pino), (USNM-177663, NMNH database). Espécimen cazado por B. V. Lilly documentado por Carmony (1998).

15/7/1911, San Luis Mountains, 55 Mi S Of Us Boundary, Agua Prieta, +, Cráneo, B. V. Lilly, 1904 msnm, pastizal natural (incluye pastizal-huizachal), (USNM-177662, NMNH database), especimen cazado por B. V. Lilly documentado por Carmony (1998).

21/8/2001, Rancho El Temor, Sierra de Huachinera, Bacadéhuachi, đ̃, Cráneo, J. P. Gallo Reynoso, 1700 msnm, bosque de encino, (CIAD-210801-1, CIAD database, Gallo et al. 2008). Cráneo donado por los rancheros, cazado con cepo.

21/8/2001, Rancho Los Huíjolos, Sierra de Huachinera, Huachinera, ㅇ, Cráneo, J. P. Gallo Reynoso, 1506 msnm, bosque de encino, (CIAD-210801-3, CIAD database, Gallo et al. 2008). Cráneo con un balazo en la cabeza, donado por los rancheros.

21/8/2001, Rancho Los Huíjolos, Sierra de Huachinera, Huachinera, ㅇ, Cráneo, J. P. Gallo Reynoso, 1665 msnm, bosque de encino, (CIAD-210801-2, CIAD database, Gallo et al. 2008). Cráneo donado por los rancheros, cazado con cepo.

0/10/2004, Rancho El Pinito, Sierra de San Luis, Agua Prieta, Observado, C. A. López-González, 1805 msnm, bosque de pino-encino (incluye encino-pino), (Com. Pers. Dr. Carlos A. López-González).

12/6/2005, La Estancia, Cumpas, $\partial$, Observado \& fotografiado, $1621 \mathrm{msnm}$, bosque de encino, (Periódico El Imparcial 2008). En el 2008, en el periódico de distribución local en Aconchi, Sonora se reporta con una foto, la intrusión de un macho al subadulto que bajo de la sierra.

0/4/2007, Rancho El Pinito, Sierra de San Luis, Agua Prieta, Excreta, C. Moreno-Arzate, 1556 msnm, bosque bajo-abierto, (Moreno-Arzate 2007).

0/4/2007, Rancho El Pinito, Sierra de San Luis, Agua Prieta, Excreta, C. Moreno-Arzate, 1805 msnm, bosque de pino, (Moreno-Arzate 2007).

0/10/2007, Rancho El Pinito, Sierra de San Luis, Agua Prieta, Excreta, C. Moreno-Arzate, 1600 msnm, bosque de pino-encino (incluye encino-pino), (Moreno-Arzate 2007).

0/10/2007, Rancho El Pinito, Sierra de San Luis, Agua Prieta, Excreta, C. Moreno-Arzate, $2271 \mathrm{msnm}$, bosque de pino-encino (incluye encino-pino), (Moreno-Arzate 2007).

0/10/2009, Rancho El Tápila, Sierra de San Luis, Agua Prieta, Fotografía, N. Lara-Díaz, 1519 msnm, bosque de pino-encino (incluye encino-pino), (Lara-Díaz 2010).

0/4/2009, Sierra de Ajos y Buenos Aires Fracción 4, Fronteras, Fotografía, N. Lara-Díaz, 2064 msnm, bosque de pino-encino (incluye encino-pino), (Lara-Díaz 2010).

0/5/2009, W Sierra Madera, Cumpas, Fotografía, N. Lara-Díaz, 1173 msnm, matorral subtropical, (LaraDíaz 2010).

0/6/2009, Sierra del Tigre Fracción 1, Nacozari de García, Fotografía, N. Lara-Díaz, 2057 msnm, bosque de encino, (Lara-Díaz 2010). 
0/7/2009, Sierra de San Diego Fracción 3, Bavispe, Fotografía, N. Lara-Díaz, 1902 msnm, bosque de pino-encino (incluye encino-pino), (Lara-Díaz 2010).

0/9/2009, Rancho Los Ojos, Sierra de San Luis, Agua Prieta, Fotografía, N. Lara-Díaz, 1631 msnm, bosque de encino, (Lara-Díaz 2010).

26/6/2010, Rancho El Pinito, Sierra de San Luis, Agua Prieta, Fotografía, N. Lara-Díaz \& C. A. LópezGonzález, 1808 msnm, pastizal natural (incluye pastizal-huizachal), (UAQ-S/Cat, UAQ database, López-González \& Lara-Díaz 2010). Registros obtenidos de un proyecto de la UAQ, financiado por el Instituto Nacional de Ecología (INE).

30/6/2010, Rancho El Pinito, Sierra de San Luis, Agua Prieta, Fotografía, N. Lara-Díaz \& C. A. LópezGonzález, 1600 msnm, bosque de pino-encino (incluye encino-pino), (UAQ-S/Cat, UAQ database, López-González \& Lara-Díaz 2010). Registros obtenidos de un proyecto de la UAQ, financiado por el Instituto Nacional de Ecología (INE).

1/7/2010, Rancho El Pinito, Sierra de San Luis, Agua Prieta, Fotografía, N. Lara-Díaz \& C. A. LópezGonzález, 1887 msnm, bosque de pino-encino (incluye encino-pino), (UAQ-S/Cat, UAQ database, López-González \& Lara-Díaz 2010). Registros obtenidos de un proyecto de la UAQ, financiado por el Instituto Nacional de Ecología (INE).

3/8/2010, Sierra de San Luis, Agua Prieta, Fotografía, N. Lara-Díaz \& C. A. López-González, 1401 msnm, bosque bajo-abierto, (UAQ-S/Cat, UAQ database, López-González \& Lara-Díaz 2010). Registros obtenidos de un proyecto de la UAQ, financiado por el Instituto Nacional de Ecología (INE).

7/8/2010, Sierra de San Luis, Agua Prieta, Fotografía, N. Lara-Díaz \& C. A. López-González, 1321 msnm, bosque bajo-abierto, (UAQ-S/Cat, UAQ database, López-González \& Lara-Díaz 2010). Registros obtenidos de un proyecto de la UAQ, financiado por el Instituto Nacional de Ecología (INE).

9/8/2010, Sierra de San Luis, Agua Prieta, Fotografía, N. Lara-Díaz \& C. A. López-González, 1402 msnm, bosque bajo-abierto, (UAQ-S/Cat, UAQ database, López-González \& Lara-Díaz 2010). Registros obtenidos de un proyecto de la UAQ, financiado por el Instituto Nacional de Ecología (INE).

28/8/2010, Sierra de San Luis, Agua Prieta, Fotografía, N. Lara-Díaz \& C. A. López-González, 1539 msnm, bosque bajo-abierto, (UAQ-S/Cat, UAQ database, López-González \& Lara-Díaz 2010). Registros obtenidos de un proyecto de la UAQ, financiado por el Instituto Nacional de Ecología (INE).

11/10/2010, Sierra de San Luis, Agua Prieta, Fotografía, N. Lara-Díaz \& C. A. López-González, 1497 msnm, bosque bajo-abierto, (UAQ-S/Cat, UAQ database, López-González \& Lara-Díaz 2010). Registros obtenidos de un proyecto de la UAQ, financiado por el Instituto Nacional de Ecología (INE).

19/10/2010, Rancho El Pinito, Sierra de San Luis, Agua Prieta, Fotografía, N. Lara-Díaz \& C. A. LópezGonzález, $1520 \mathrm{msnm}$, bosque de pino-encino (incluye encino-pino), (UAQ-S/Cat, UAQ database, López-González \& Lara-Díaz 2010). Registros obtenidos de un proyecto de la UAQ, financiado por el Instituto Nacional de Ecología (INE).

28/10/2010, Sierra de San Luis, Agua Prieta, Excreta, N. Lara-Díaz \& C. A. López-González, 1610 msnm, pastizal natural (incluye pastizal-huizachal), (UAQ-S/Cat, UAQ database, López-González \& Lara-Díaz 2010). Registros obtenidos de un proyecto de la UAQ, financiado por el Instituto Nacional de Ecología (INE).

30/10/2010, Sierra de San Luis, Agua Prieta, Excreta, N. Lara-Díaz \& C. A. López-González, 1616 ms$\mathrm{nm}$, bosque de encino, (UAQ-S/Cat, UAQ database, López-González \& Lara-Díaz 2010). Registros obtenidos de un proyecto de la UAQ, financiado por el Instituto Nacional de Ecología (INE).

3/11/2010, Sierra de San Luis, Agua Prieta, Huella, N. Lara-Díaz \& C. A. López-González, 1519 msnm, bosque de pino-encino (incluye encino-pino), (UAQ-S/Cat, UAQ database, López-González \& Lara-Díaz 2010). Registros obtenidos de un proyecto de la UAQ, financiado por el Instituto Nacional de Ecología (INE). 